\title{
Dissipation Characteristics of Tornadic Vortex Signatures Associated with Long-Duration Tornadoes
}

\author{
MiCHAEL M. FRENCH \\ School of Marine and Atmospheric Sciences, Stony Brook University, Stony Brook, New York \\ DARREL M. KINGFIELD \\ Cooperative Institute for Research in Environmental Sciences, University of Colorado, and NOAA/Office of Oceanic \\ and Atmospheric Research/Earth System Research Laboratory Global Systems Division, Boulder, Colorado
}

(Manuscript received 17 July 2018, in final form 6 December 2018)

\begin{abstract}
Weather Surveillance Radar-1988 Doppler (WSR-88D) data from 36 tornadic supercell cases from 2012 to 2016 are investigated to identify common tornadic vortex signature (TVS) behaviors prior to tornado dissipation. Based on the results of past case studies, four characteristics of TVSs associated with tornado dissipation were identified: weak or decreasing TVS intensity, rearward storm-relative motion of the TVS, large or increasing TVS vertical tilt, and large or increasing TVS horizontal displacement from the main storm updraft. Only cases in which a TVS was within $60 \mathrm{~km}$ of a WSR-88D site in at least four consecutive volumes at the end of the tornado life cycle were examined. The space and time restrictions on case selection ensured that the aforementioned quantities could be determined within $\sim 500 \mathrm{~m}$ of the surface at several time periods despite the relatively coarse spatiotemporal resolution of WSR-88D systems. It is found that prior to dissipation, TVSs become increasingly less intense, tend to move rearward in a storm-relative framework, and become increasingly more separated from the approximate location of the main storm updraft. There is no clear signal in the relationship between tornado tilt, as measured in inclination angle, and TVS dissipation. The frequency of combinations of TVS dissipation behaviors, the impact of increased low-level WSR-88D scanning on dissipation detection, and prospects for future nowcasting of tornado life cycles also are discussed.
\end{abstract}

\section{Introduction}

A pioneering advancement in tornado science occurred with the discovery of the tornadic vortex signature (TVS; Brown et al. 1978), a bulk representation of a tornado that appears as a distinctive small-scale enhancement in azimuthal shear in Doppler radar data. Most tornadoes are not spatially well resolved by the radar, so a TVS is larger than the actual tornado (Brown et al. 1978) and, owing to finite radar sampling, debris effects, and beam spreading, among other limitations, a TVS contains radial velocities that are less intense than wind speeds in the tornado near the surface (Snyder and Bluestein 2014). Nevertheless, the TVS serves as a useful proxy for the presence of a tornado in data from the Weather Surveillance Radar-1988 Doppler (WSR-88D)

Corresponding author: Michael M. French, michael.m.french@ stonybrook.edu network. In addition, the polarimetric tornadic debris signature (TDS; Ryzhkov et al. 2005) is commonly associated with tornadoes to the point that tornado detection can be partially automated (Snyder and Ryzhkov 2015). Therefore, given a sufficiently short distance and clear line of sight between the radar and the tornado, identification of existing tornadoes in Doppler weather radar data is nearly ubiquitous. The TVS and TDS serve as useful tornado proxies both for forecasters issuing warnings (e.g., Blair and Leighton 2014) and for researchers conducting observational studies (e.g., Trapp et al. 1999).

One focus of radar-based observational research into tornado processes has been on determining how, and in what manner, tornadoes form (e.g., Brandes 1977, 1978, 1981; Lemon and Doswell 1979; Dowell and Bluestein 2002a,b; Bluestein et al. 2003; Wurman et al. 2007a; Markowski et al. 2012; French et al. 2013: Kosiba et al. 2013; Houser et al. 2015) and/or why tornadoes are 
favored within some supercells and not others (e.g., Markowski et al. 2011; Klees et al. 2016). In addition to tornadogenesis being a largely unsolved scientific problem (e.g., Markowski and Richardson 2009), the inability to consistently discriminate between tornadic and nontornadic storms impedes operational meteorologists from being able to skillfully nowcast whether or not a tornado will form in order to mitigate harm to life and property [e.g., see Fig. 1 in Wurman et al. (2012)]. The source of the nowcasting problem goes beyond the gap in tornadogenesis knowledge in that conditions and processes that are known to be supportive of tornadogenesis do not have "fingerprints" that are easily observable for forecasters in real time. Examples include positively buoyant rear-flank downdraft outflow (Markowski et al. 2002), generation of vertical vorticity in downdrafts via a "slippage" mechanism (DaviesJones and Brooks 1993; Dahl et al. 2014), and low-level, dynamically driven updrafts that support tornado-scale vorticity tilting and stretching (e.g., Markowski and Richardson 2014).

Another open research question that has received comparatively less attention in the literature is the storm-scale or sub-storm-scale processes that lead to tornado dissipation. As with tornadogenesis, the motivation for this research is not only to understand why tornadoes dissipate, but also to use that knowledge to inform the accurate issuance and expiration of tornado warnings. An improved ability to nowcast tornado dissipation would be particularly useful for "long track" tornadoes that require multiple warnings, and for optimizing information about tornado life cycles to first responders and others tasked with entering areas affected by tornadoes as rapidly and safely as possible. However, unlike with nowcasting tornadogenesis, nowcasting tornado dissipation can involve assessing known fingerprints in near-real time by tracking the behavior of the TVS itself.

Determining how and why tornado dissipation occurs is an open problem that has been commonly investigated using individual case studies. For a review of the current knowledge regarding vortex dissipation within the cyclic tornadogenesis process (Darkow and Roos 1970), see Burgess et al. (1982), Adlerman et al. (1999), and Dowell and Bluestein (2002a,b). For a thorough review of potential causes of tornado dissipation, including those outside of the cyclic tornadogenesis process, see Marquis et al. (2012). A discussion of the various outstanding questions regarding the topic is beyond the scope of this paper. Instead, we aim to seek out specific effects of dissipation processes that are identifiable in radar data from the WSR-88D network in near-real time.
Based on our interpretation of past studies, we have identified four behaviors of TVSs ${ }^{1}$ that may be associated with an increased likelihood of tornado dissipation: 1) weak or decreasing TVS "intensity", 2) rearward stormrelative (SR) motion of the TVS, 3) large or increasing TVS vertical tilt, and 4) large or increasing TVS horizontal displacement from the main storm updraft. These four TVS behaviors do not constitute the only observational indicators of tornado dissipation; rather, they are the ones we believe to be supported for study by past literature, detectable using WSR-88D data, and amenable for possible use in future nowcasting algorithms. A brief summary justifying these choices follows.

There is considerable evidence that tornadoes have defined life cycles in which they weaken at the surface before they dissipate. This claim is supported by results from damage surveys (e.g., Wakimoto et al. 2003; Atkins et al. 2014) and dual-Doppler retrievals of vertical vorticity (Brandes 1984; Dowell and Bluestein 2002a; Marquis et al. 2012). In addition, there is evidence that the low-level TVS associated with the tornado in mobile Doppler radar data will reflect this defined life cycle via $\Delta V$ values (maximum sum of inbound and outbound radial velocities within the TVS) that decrease (e.g., Fig. 1a) prior to tornado dissipation (e.g., Wurman and Gill 2000; Wurman et al. 2007a,b; 2010; Marquis et al. 2008; Kosiba et al. 2013; French et al. 2014; Houser et al. 2015; Bluestein et al. 2016). However, TVS intensity in coarse-resolution WSR-88D data is unlikely to reflect the strength of tornadic winds (e.g., Brown et al. 1978; Burgess et al. 1993; Wood and Brown 1997; Trapp et al. 1999; Kingfield and LaDue 2015). In addition, tornado weakening is sometimes observed prior to reintensification and followed by prolonged tornado persistence (e.g., Kurdzo et al. 2015) or the weakening is unsteady and/or abrupt (e.g., Davies-Jones et al. 1978; Burgess et al. 2002; Wurman et al. 2010). As a result, one evident area to explore is the magnitude of TVS $\Delta V$ and its changes in the minutes leading up to the approximate time of tornado dissipation.

The second behavior, SR rearward TVS motion, also is consistently documented in past studies (e.g., Fig. 1b). Movement of the tornado rearward and to the left of supercell updraft/storm motion shortly before dissipation has been documented in numerous studies (Fujita et al. 1970; Burgess et al. 1982; Dowell and Bluestein 2002a; Speheger et al. 2002; Tanamachi et al. 2012) and is thought to result from advection by the SR

\footnotetext{
${ }^{1}$ Investigation of changes to TDS characteristics prior to tornado dissipation was not undertaken in this study because there is evidence that TDS variability is largely driven by changes in tornado intensity (e.g., Bodine et al. 2013), which makes its study redundant with using radial velocity to estimate intensity via $\Delta V$ calculations.
} 
flow within the cyclic tornadogenesis process (Dowell and Bluestein 2002b). In addition, French et al. (2014) used data from two cases to hypothesize that tornado dissipation may be preceded by SR tornado motion to the right of the storm in cases in which there is large rearflank gust-front outflow and weak storm inflow, which would be associated with storms not exhibiting cyclic tornadogenesis. Thus, we investigate whether SR rearward motion is commonly associated with tornado dissipation, and if the cyclic tornadogenesis process is associated with lateral SR motion.

A commonly observed feature of tornadoes is that their vertical tilt varies within their life cycles. Upon discovery of the TVS, initial radar observations were of a TVS tilted up to $45^{\circ}$ (Davies-Jones 1982) and to the left of the storm motion vector (Brown et al. 1978). Subsequent observations from both fixed-site and mobile Doppler radars found TVSs that tilted toward the northeast or northwest with increasing height (Wakimoto and Martner 1992; Wurman and Gill 2000, Lee and Wurman 2005; Alexander and Wurman 2005; Tanamachi et al. 2012; French et al. 2014; Houser et al. 2015; Bluestein et al. 2016). The radar observations are consistent with visual sightings of tornado tilting in the subcloud layer during the dissipation stage (e.g., Golden and Purcell 1977, 1978; Bluestein et al. 1988). Only recently in French et al. (2014) has a time series of tornado inclination angles (i.e., the angle from the vertical between the TVS at two levels) been documented quantitatively to determine changes in tornado tilt. It was found that large increases in inclination angle preceded TVS dissipation for two cases (e.g., Fig. 1c), likely from outflow-induced differential horizontal advection of the tornado. Owing to the results of that study and ubiquitous observations of tilted dissipating tornadoes, we investigate whether large and/ or increasing tornado inclination angle from the surface to low levels [ $\sim 2-3 \mathrm{~km}$ above radar level (ARL); hereafter all heights are ARL] in time is associated with tornado dissipation.

A fourth behavior that may be associated with tornado dissipation is large or increasing horizontal displacement between the tornado at low levels and the midlevel storm updraft/mesocyclone. Results from numerical simulations (Wicker and Wilhelmson 1995; Guarriello et al. 2018), high-resolution single-Doppler (Wurman et al. 2010; Skinner et al. 2014), dual-Doppler (Wakimoto and Martner 1992), and ensemble Kalman filter (Marquis et al. 2012) analyses have documented tornado or lowlevel vortex maintenance disruption as the vortex becomes increasingly displaced from the midlevel mesocyclone (e.g., Fig. 1d); this misalignment is likely to lessen dynamically driven updrafts that enhance vertical vorticity stretching and contribute to vortex formation and maintenance (e.g., Markowski and Richardson 2014). Alignment of low- and midlevel circulations has not been the focus of many mobile Doppler radar case studies because of the tendency for their data collection to focus on storm low levels. The use of WSR-88D data provides an opportunity to thoroughly investigate the relationship between TVS-midlevel updraft horizontal displacement and tornado dissipation.

In this paper, we aim to move beyond individual case studies in determining, in a bulk sense, what TVS behaviors are associated with tornado dissipation. To do so, a sample of tornadic supercell cases were examined using WSR-88D data with strict thresholds for range and duration to ensure a sufficient number of data points and appropriate height levels. In section 2, the cases chosen and WSR-88D data used are described. In section 3, the four TVS behaviors are inspected to determine their relationship to TVS life cycles as a proxy for tornado dissipation. In section 4, the results are discussed in the context of past work and their potential for use in tornado nowcasting in the future.

\section{Data and methods}

\section{a. Case selection}

There were two main sources of data used in this study: Storm Data and level-II WSR-88D data from 2012 to 2016. A "first guess" at potential tornado cases to include in this study was provided by employing two restrictions on the former data source. First, tornado cases must have tornado duration times of at least $18 \mathrm{~min}$ in Storm Data, which was determined by using the tornado begin and end times, ${ }^{2}$ and accounting for tornadoes that traverse multiple counties. In addition, the cases must have at least $18 \mathrm{~min}$ of continuous nearsurface TVS data (i.e., a TVS in the lowest $\sim 500 \mathrm{~m}$ ) at the end of the tornado's life cycle within $60 \mathrm{~km}$ of a WSR-88D site. The restriction on the estimated duration of the tornado was enlisted so that each case would have at least four radar volumes in which a TVS was identified (radar volume update times are a minimum of $\sim 4.5 \mathrm{~min}$ ). Cases with at least four volumes were then used not only to determine characteristics of the TVSs, but also to estimate how those characteristics changed from volume to volume leading up to dissipation. The range restriction was established so that data at a height of $\sim 500 \mathrm{~m}$ were available in each case.

\footnotetext{
${ }^{2}$ Severe weather begin and end times in Storm Data likely include significant errors (e.g., Witt et al. 1998); its use was only to identify a subset of potential long-duration tornado cases, among a full set of thousands, for further evaluation.
} 


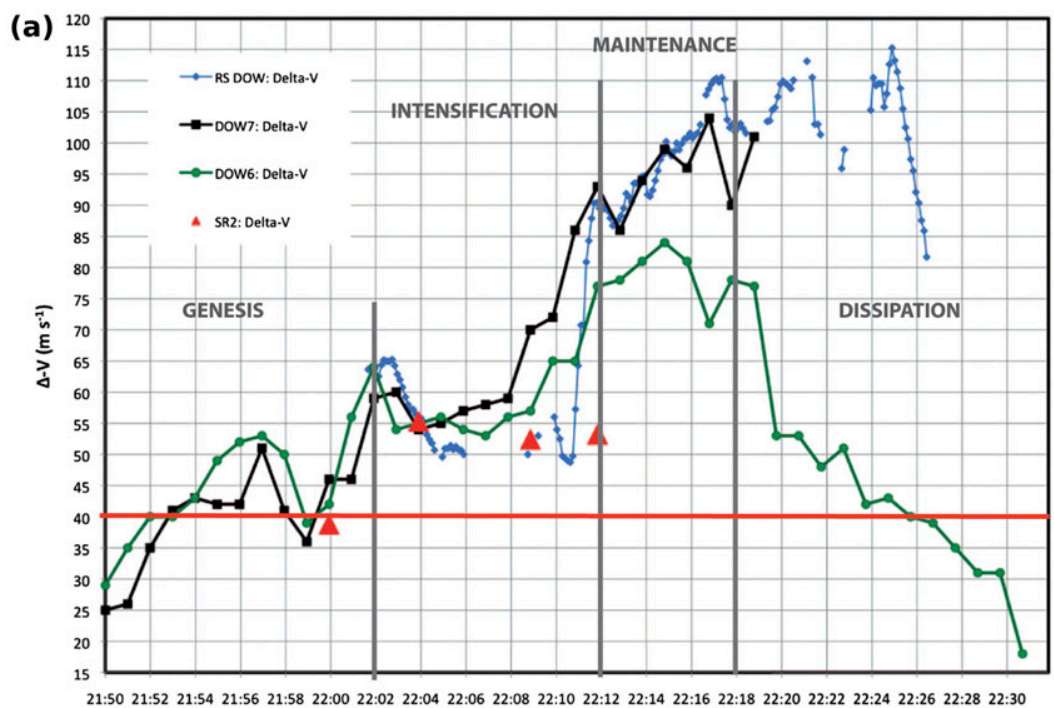

(b)

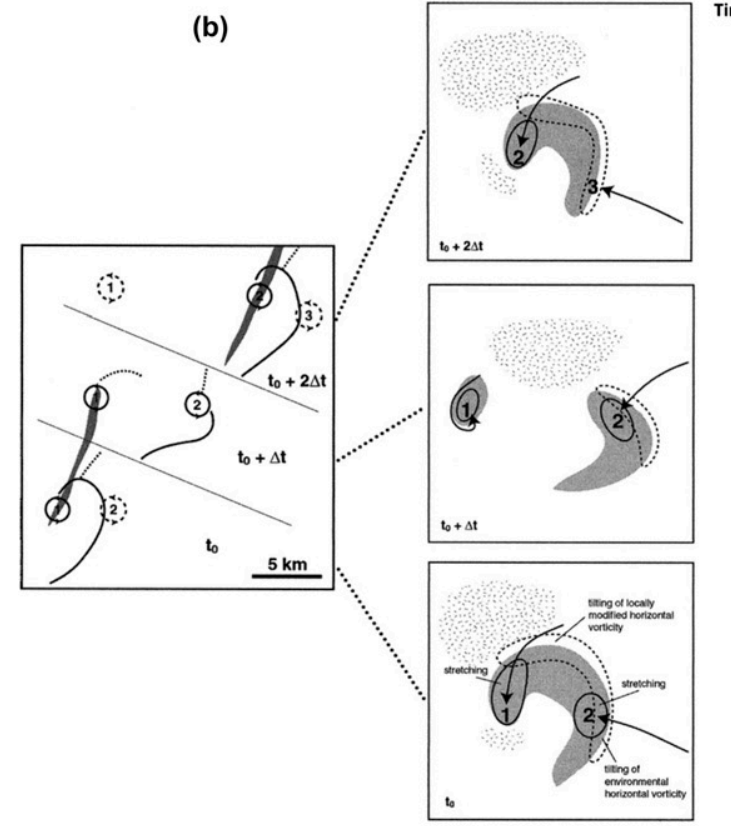

(d)
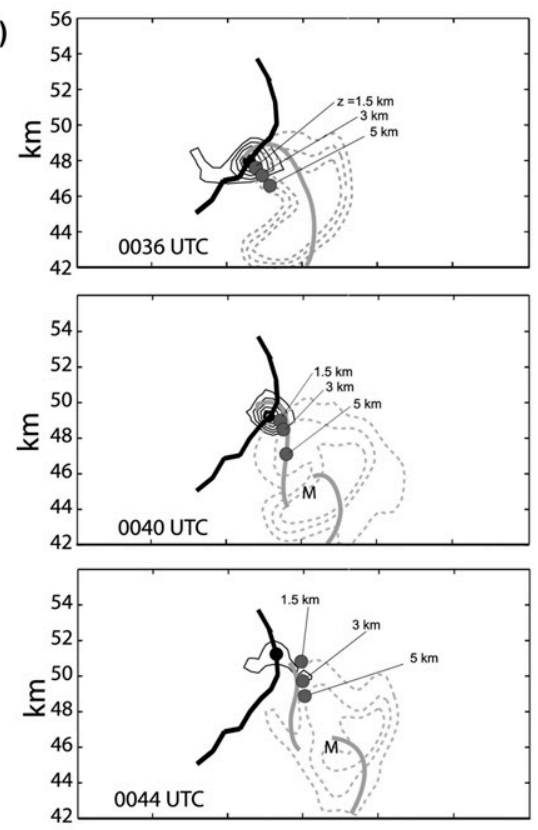

(c)

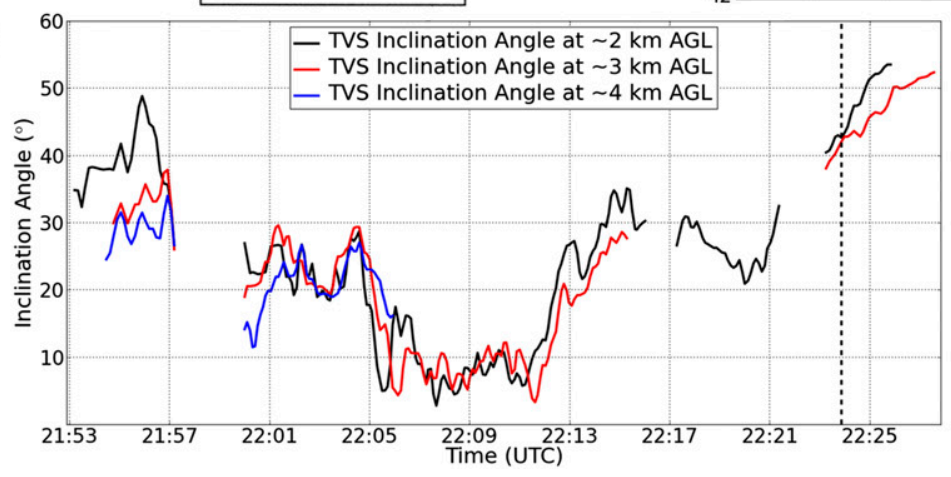

FIG. 1. Evidence from past case studies of radar behaviors identified as being associated with tornado dissipation: (a) Time series of approximated TVS intensity (low-level $\Delta V$ ) showing weakening before TVS dissipation from Doppler on Wheels 6 (DOW6; green line) and the Rapid-Scan DOW (blue line) for the Goshen County tornado on 5 Jun 2009. The horizontal red line marks a subjective TVS $\Delta V$ cutoff for a tornado. From Kosiba et al. (2013). (b) Conceptual model of the cyclic tornadogenesis process showing rearward storm-relative motion prior to TVS 

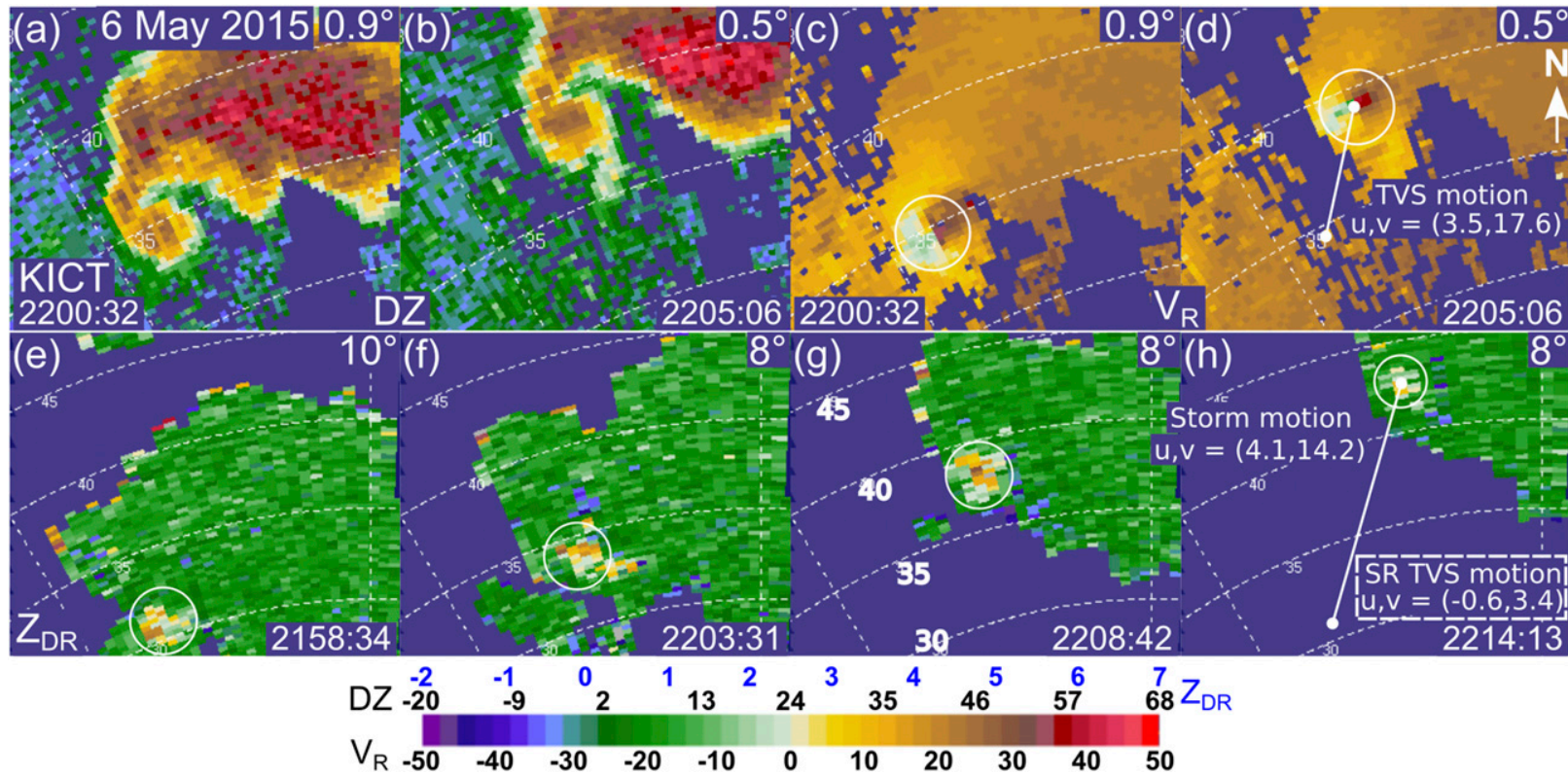

FIG. 2. An illustration of the TVS motion, storm motion, and storm-relative TVS motion estimation process using data from the KICT WSR-88D on 6 May 2015, one of the cases analyzed in this study. (a),(b) Reflectivity factor (dBZ) at 2200:32 and 2205:06 UTC, respectively, (c),(d) radial velocity $\left(\mathrm{m} \mathrm{s}^{-1}\right)$ at 2200:32 and 2205:06 UTC, respectively, and (e)-(h) differential radar reflectivity factor (dB) at 2158:34, 2203:31, 2208:42, and 2214:13 UTC, respectively. Tornado motion between successive volumes is estimated using the position of the TVS in (c) and (d). Storm motion is estimated by tracking the mid- or upper-level $Z_{\mathrm{DR}}$ column in the storm over a 15 -min period centered on the period of time between volumes in (e) and (h). Storm-relative TVS motion is then determined by subtracting storm motion components from TVS motion components. The relevant $u$ and $v$ components of motion ( $\mathrm{m} \mathrm{s}^{-1}$ ) are provided in (d) and (h). Range rings are every $5 \mathrm{~km}$ and are labeled in (g) for convenience. The approximate center radar beam heights at the locations of the TVS are $\sim 550$ and $350 \mathrm{~m}$ ARL for (c) and (d), respectively, and of the $Z_{\mathrm{DR}}$ column centroid are $\sim 5.6,4.8,5.2$, and 5.9 km ARL for (e)-(h), respectively.

If the Storm Data conditions were met, the WSR-88D traditional moment and polarimetric data of each case were analyzed. The 2012-16 time period restriction used here ensured that all radar data were obtained 1) by employing azimuthal oversampling at low elevation angles ("super resolution" data; Torres and Curtis 2007) and 2) after the dual-polarization upgrade to the WSR88D network. Use of super-resolution data allowed for spatial sampling differences among cases to be minimized and TVS detection to be optimized (Wood et al. 2009). Polarimetric data were used to identify the approximate location of the updraft by analyzing differential radar reflectivity factor $Z_{\mathrm{DR}}$ from above the freezing level (see section 2c).

For each potential case, radar data from $15 \mathrm{~min}$ before the tornado begin time until $15 \mathrm{~min}$ after the tornado end time in Storm Data were inspected to determine whether the storm that spawned the tornado was a

dissipation as deduced from P-3 aircraft pseudo dual-Doppler analyses in the McLean, TX, supercell of 8 Jun 1995. (b) (left) Numbered circles identify tornadoes, solid lines are wind shifts, and shaded areas are tornado tracks. (b) (right) Solid gray shading indicates primary areas of vertical vorticity stretching, dotted outlines mark areas of tilting of horizontal vorticity into vertical vorticity, and arrows are vortex-relative trajectories. From Dowell and Bluestein (2002b). (c) Time series of TVS inclination angles $\left(^{\circ}\right.$ ) for the Goshen County tornado showing an increase in tornado tilt prior to tornado dissipation between the near-surface and 2 (black line), 3 (red line), and $4 \mathrm{~km}$ (blue line) above ground level. Data are smoothed with a 1-2-1 time filter and the vertical line is the estimated time of tornado dissipation. From French et al. (2014). (d) Ensemble-mean vertical velocity at $z=5 \mathrm{~km}$ AGL (gray dashed contours of $w=5,10,15 \mathrm{~m} \mathrm{~s}^{-1}$ ), vertical vorticity at $z=500 \mathrm{~m}$ AGL (thin black contours; outermost contour is $0.01 \mathrm{~s}^{-1}$, incremented by $0.005 \mathrm{~s}^{-1}$ ), the position of the surface gust fronts (traced with thick gray lines), and the surface track of the tornado (thick black line) at three times for a tornado near Almena, KS, on 3 June 1999. The centers of the mesocyclone containing the tornado located at $z=1.5,3$, and $5 \mathrm{~km}$ AGL are shown with gray dots. The M indicates the location of a newly developing surface mesocyclone. Adapted from Marquis et al. (2012). 
supercell and if there was a continuous TVS that could be tracked and interrogated continuously in its last four volumes. The storm type was determined subjectively; all cases were found to be either supercells or quasilinear convective systems (QLCSs). We examined only supercell cases in this study for two reasons: 1) the TVS behaviors investigated are based on hypotheses generated only in past supercell case studies and 2) it was found that QLCS TVSs were difficult to track, reducing the number of usable cases. Supercells were identified by the presence of a persistent midlevel mesocyclone in radial velocity data throughout the life cycle of the TVS. Radial velocities within the TVS were edited subjectively to remove velocity aliasing before assessing the signature's low-level time continuity. If there were four consecutive TVS scans leading to dissipation, the case was included in this study.

\section{b. TVS identification}

The major source of analysis in this study was the identification of TVSs and the calculation of several of their characteristics. Objective TVS criteria have varied depending on the Doppler radar being used (e.g., Trapp et al. 1999; Alexander 2010; French et al. 2013) and how one defines a tornado (Tanamachi et al. 2013; Wurman and Kosiba 2013). Here, we used a rigid definition of a TVS, assuming the signature was identified within 15 min of a reported tornado in Storm Data (e.g., Witt et al. 1998): there must be local inbound and outbound radial velocity maxima with a difference between them $\Delta V$ of at least $15 \mathrm{~m} \mathrm{~s}^{-1}$ and separated by a distance $\mathrm{e}^{3}<1.5 \mathrm{~km}$ at the lowest elevation angle with noncontaminated data. A local maximum required there to be no radar gate adjacent to the gate in question with a radial velocity magnitude at least $1.0 \mathrm{~m} \mathrm{~s}^{-1}$ greater than the maximum value (i.e., if adjacent azimuths had nearly the same values, the radar gate closest to the center of the circulation was used in the calculations). The $15 \mathrm{~m} \mathrm{~s}^{-1} \Delta V$ cutoff is the same as that used in Trapp et al. (1999) for WSR-88D data and reflects the use of data at ranges as great as $60 \mathrm{~km}$ and, therefore, relatively large volumes that increasingly underestimate the true intensity of the tornado (Brown et al. 1978).

The distance threshold between incoming and outgoing maxima was used to minimize the impact that misidentified mesocyclones would have on contaminating the results. In addition, TVS peaks tend to be separated by one radar beamwidth regardless of tornado size, strength, or structure (Wood and Brown 2011) so that a

\footnotetext{
${ }^{3}$ If the midpoint between two or more gates was $<1.5 \mathrm{~km}$, the next gate was permitted for analysis.
}

1.5-km distance should account for virtually all legitimate observations. It was common aloft (heights $>2 \mathrm{~km}$ ) for there to be a mesocyclone-scale indication of rotation but no radar indication of a smaller-scale circulation. Either there was no tornado at these levels despite there being one near the surface (e.g., French et al. 2014) or a TVS could not be distinguished from the mesocyclone (e.g., Brown and Wood 2015). We erred on the side of excluding TVS data in these situations so as to prevent incorporation of mesocyclone data into the analysis. Gaps in TVS identification aloft (i.e., at levels $>500 \mathrm{~m}$ ) were permitted, but cases in which the TVS at $\sim 500 \mathrm{~m}$ was not identified in at least four consecutive volumes up to dissipation were not included in this study.

To this point, we have only discussed the TVS and not the tornado signature (TS). A TVS is different from a radar TS in that the former (latter) requires the core diameter of the tornado to be less (greater) than the radar's effective beamwidth (Brown 1998). In this study, both TVSs and TSs were identified and used interchangeably in the analysis. Hereafter, for convenience and to prevent confusion, we use TVS to refer to all of the radar vortex signatures assumed to represent the tornado in radial velocity data.

\section{c. Updraft location and storm motion}

In the absence of retrieved vertical velocity data, determination of storm motion is often inexact and fraught with potential problems, owing to difficulty identifying the location of the main storm updraft. In past singleDoppler radar studies, storm motion was determined by following features in radar reflectivity like a storm "vault" (e.g., Tanamachi et al. 2012) or forward-flank reflectivity maxima (e.g., French et al. 2008). In this study, we instead used a methodology that we believe is more consistent in tracking the storm updraft: using the centroid of the $Z_{\mathrm{DR}}$ column at the highest level in which the feature was identified and easy to track. It has been shown in several studies, both observational and modeling, that the $Z_{\mathrm{DR}}$ column is adjacent to or collocated with the storm updraft (Kumjian and Ryzhkov 2008), as large raindrops and small hail are being lofted by its strong vertical velocities above the freezing level (Illingworth et al. 1987). More recent studies have even shown a direct relationship between $Z_{\mathrm{DR}}$ column height and vertical velocity in the updraft (Kumjian et al. 2014).

The first step in determining storm motion was to identify the approximate location of the updraft via the $Z_{\mathrm{DR}}$ column in each radar volume. At successive levels above the freezing level (in which the values of $Z_{\mathrm{DR}}$ are $\sim 0 \mathrm{~dB}$ for most of the storm indicating the dominant presence of dry snow), the location of an 
area of $Z_{\mathrm{DR}} \geq 1.0 \mathrm{~dB}$ coincident with the supercell thunderstorm's expected updraft location (i.e., overlapping with broad cyclonic shear in radial velocity, reduced copolar correlation coefficient, and reduced reflectivity factor) was documented. The threshold $Z_{\mathrm{DR}}$ value of $1.0 \mathrm{~dB}$ is based on past studies that have focused on identifying $Z_{\mathrm{DR}}$ columns (Snyder et al. 2015). The highest scan at which this enhanced $Z_{\mathrm{DR}}$ area was small enough to isolate from any contribution of enhanced $Z_{\mathrm{DR}}$ from other sources, yet large enough to track easily over several volumes, was used. The centroid of the continuous $Z_{\mathrm{DR}}$ area then was approximated using the mean $x$ and $y$ coordinates and recorded. This process was repeated for each radar volume in which a TVS was identified, and also two volumes pre-TVS and one volume post-TVS. The interrogation of $Z_{\mathrm{DR}}$ only above the freezing level mitigated any concerns about differential attenuation; none of the cases used in this study had identifiable polarimetric data quality issues.

Both the zonal and meridional components of the storm motion vector were approximated by using the distance between the updraft locations and the corresponding times between volumes. To reduce the impact of storm motion errors owing to subjectivity involved in locating the center of the $Z_{\mathrm{DR}}$ column, storm motion calculations for each volume used a window of $\sim 15 \mathrm{~min}$ of time beginning (ending) with the volume two previous (one subsequent) to the volume in question. Therefore, the storm motion is more accurately the average updraft motion during a period of time centered on the $\sim 5$-min period prior to the volume in question. The use of the $\sim 15$-min period means that, given $Z_{\mathrm{DR}}$ column diameters of $\sim 5 \mathrm{~km}$, an egregious $2.5-\mathrm{km}$ error in estimated column center would result in a storm motion magnitude error of $\sim 2.75 \mathrm{~m} \mathrm{~s}^{-1}$. An example of the storm motion estimation process is shown in Fig. 2. A supercell (e.g., Figs. 2a,b) that produced a tornado identifiable as a TVS moving north-northeast (Figs. 2c,d) has its storm motion estimated also as north-northeast by tracking the $Z_{\mathrm{DR}}$ column (Figs. 2e-h); the SR TVS motion is computed by subtracting the storm motion from the TVS motion (Fig. 2h).

\section{Observations of TVS dissipation}

For this study, 36 cases $^{4}$ fit the criteria discussed in section $2 \mathrm{a}$ (Table 1). The cases vary in duration,

\footnotetext{
${ }^{4}$ In two cases, we could not identify a consistent $Z_{\mathrm{DR}}$ column to estimate storm motion and the location of the midlevel updraft; rather than applying a different methodology for just two cases, the cases were instead omitted from the SR motion and horizontal displacement aspects of the study.
}

TABLE 1. A list of the 36 tornado cases used in this study, including the date of the event, the WSR-88D site used, the EF-scale rating, and the duration of the tornado according to Storm Data.

\begin{tabular}{llcc}
\hline \hline \multicolumn{1}{c}{ Date } & $\begin{array}{c}\text { Closest } \\
\text { WSR-88D }\end{array}$ & $\begin{array}{c}\text { Damage } \\
\text { rating }\end{array}$ & $\begin{array}{c}\text { Tornado } \\
\text { duration }(\mathrm{min})\end{array}$ \\
\hline 3 Mar 2012 & KHTX & EF2 & 50 \\
15 Apr 2012 & KICT & EF3 & 30 \\
15 Apr 2012 & KVNX & EF0 & 26 \\
1 May 2012 & KVNX & EF1 & 54 \\
18 Mar 2013 & KFFC & EF2 & 20 \\
19 May 2013 & KTLX & EF3 & 50 \\
20 May 2013 & KTLX & EF4 & 39 \\
31 May 2013 & KTLX & EF3 & 41 \\
10 Jun 2013 & KHPX & EF2 & 29 \\
17 Nov 2013 & KPAH & EF2 & 21 \\
28 Apr 2014 & KLZK & EF3 & 56 \\
28 Apr 2014 & KGWX & EF2 & 22 \\
29 Apr 2014 & KHTX & EF3 & 24 \\
10 May 2014 & KEAX & EF2 & 40 \\
22 May 2014 & KENX & EF3 & 22 \\
27 Jun 2014 & KLCH & EF0 & 74 \\
7 Jul 2014 & KMQT & EF0 & 26 \\
9 Apr 2015 & KDVN & EF1 & 26 \\
6 May 2015 & KUEX & EF2 & 26 \\
6 May 2015 & KTLX & EF3 & 53 \\
6 May 2015 & KUEX & EF2 & 23 \\
6 May 2015 & KICT & EF3 & 28 \\
9 May 2015 & KDYX & EF3 & 20 \\
16 May 2015 & KFDR & EF2 & 43 \\
25 May 2015 & KDDC & EF1 & 43 \\
25 May 2015 & KDDC & EF2 & 66 \\
6 Jun 2015 & KGLD & EF0 & 45 \\
20 Jun 2015 & KUDX & EF2 & 30 \\
23 Jun 2015 & KLOT & EF3 & 27 \\
16 Nov 2015 & KDDC & EF3 & 78 \\
16 Nov 2015 & KDDC & EF1 & 24 \\
30 Mar 2016 & KINX & EF1 & 60 \\
10 May 2016 & KPAH & EF3 & 40 \\
24 May 2016 & KDDC & EF3 & 30 \\
24 May 2016 & KDDC & EF2 & 24 \\
17 Sep 2016 & KMAF & EF1 & 22 \\
\hline
\end{tabular}

enhanced Fujita scale (EF) rating, and location. The distribution of cases comprise tornadoes that are more intense than U.S. tornadoes as a whole; the large percentage of $\mathrm{EF} 2+$ cases $(\sim 70 \%)$ is consistent with the established relationship between longer tornado path lengths and stronger tornadoes (Brooks 2004). In addition, the locations of the tornado cases skew toward the southern Plains (Fig. 3), one of the preferred regions for long-track supercell tornadoes (e.g., Coleman and Dixon 2014). Therefore, the results from this study may not be representative of U.S. tornadoes as a whole because of these necessary case selection biases, and the exclusion of QLCS cases.

The cases were investigated to determine if the four TVS behaviors discussed in section 1 were associated with and/or predictors of tornado dissipation, defined 


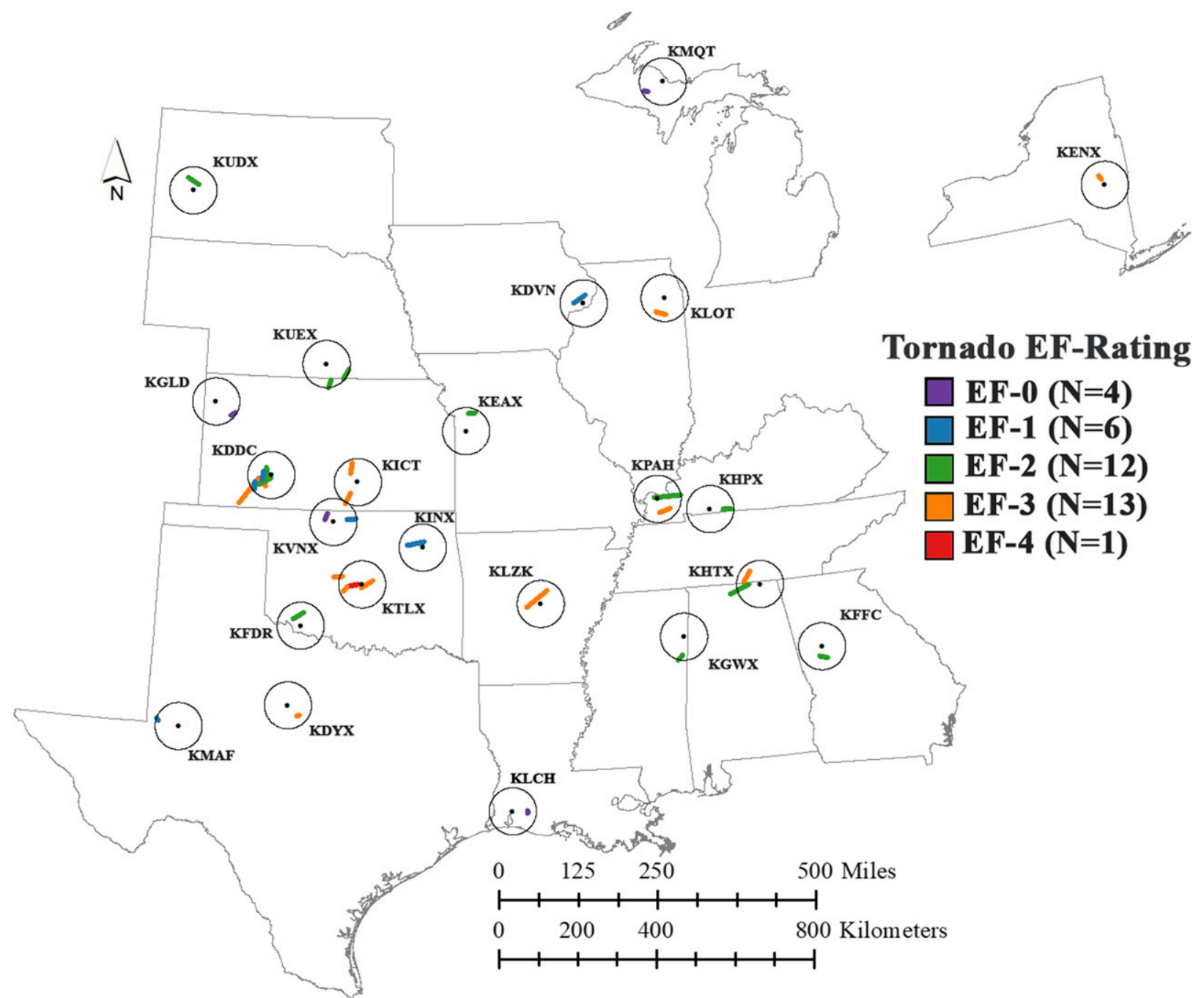

FIG. 3. Map of the long-duration tornado cases used in this study, color-coded by surveyed EF-scale rating. Also shown are the nearest WSR-88D locations and $60-\mathrm{km}$ range rings for each site.

as occurring when the aforementioned TVS criteria (section 2b) were no longer met. To best compare how TVS properties changed at similar points in the life cycles of the tornadoes, volumes were grouped relative to the last TVS observation for each case. Figures and discussion center around the final four TVS volumes for each case, labeled "D" (final TVS observation, dissipation volume), "D-1" (one volume before final), "D-2" (two volumes before final), and "D-3" (three volumes before final). Volume-to-volume changes in behaviors were normalized to 5 -min changes since the times between volumes can vary from $\sim 4.5$ to $7 \mathrm{~min}$. Additional volumes were not analyzed because sample sizes for earlier dissipation-relative times would be too low to accurately assess the significance of the results.
Statistical testing was completed using the Wilcoxon signed-rank test, a nonparametric test that determines whether paired data (in this study, one of the four dissipation behaviors from two different volumes in the same storm) have mean ranks that differ significantly from zero (Wilcoxon 1945). The use of this test avoids invoking the often dubious assumption that the underlying population is normally distributed. The version used in this study is directional (e.g., it is hypothesized that TVS intensity should be lower in the dissipation volume than in earlier volumes), applies a continuity correction, and includes zero differences in the ranking process, as proposed by Pratt (1959). Statistical significance at the $1 \%$ level $(p \leq 0.01)$ is highlighted in results; this stringent $p$ value, often deemed as identifying "very significant" statistical differences, was chosen as one 

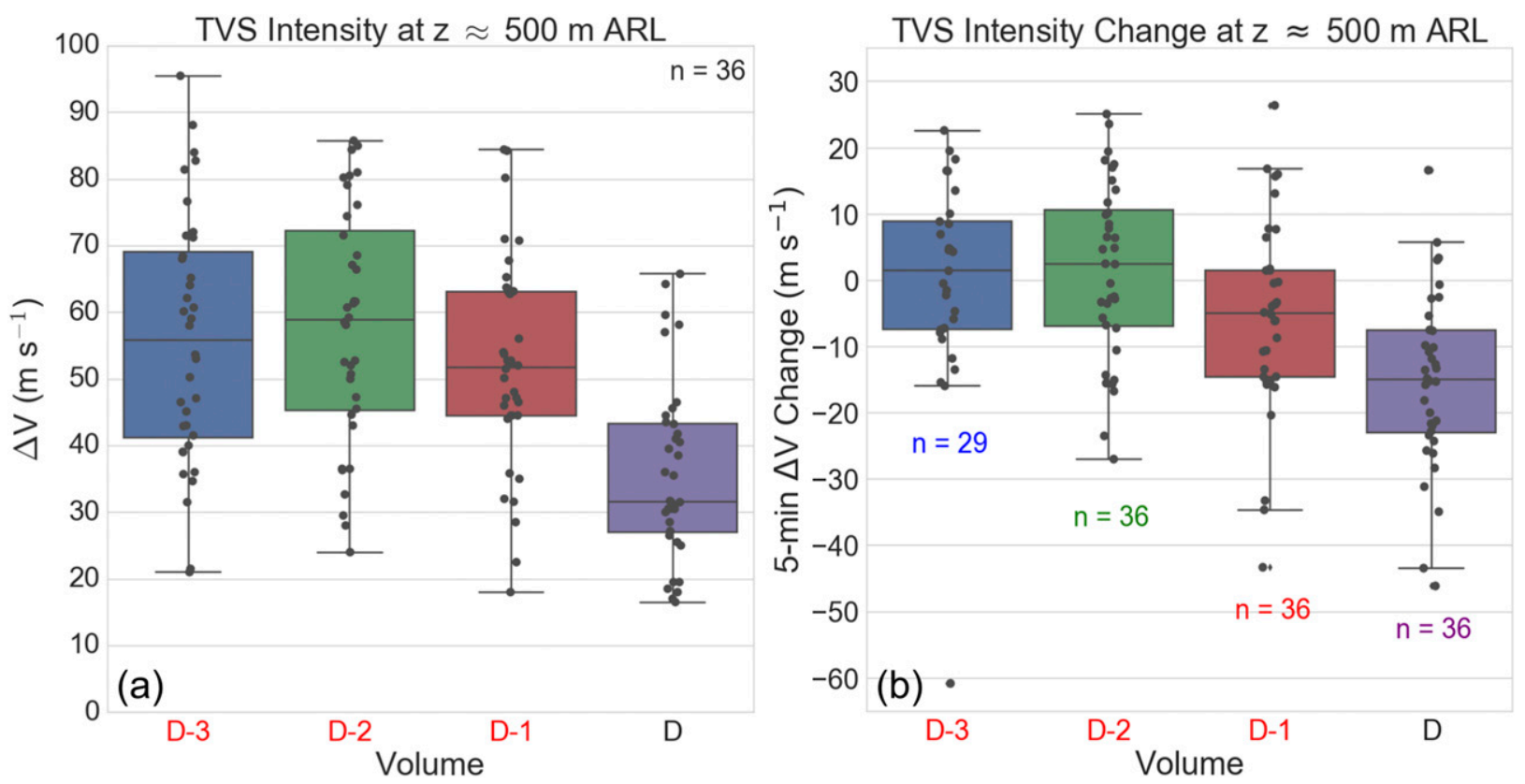

FIG. 4. Box-and-whisker plots overlaid with scatterplots showing (a) TVS $\Delta V\left(\mathrm{~m} \mathrm{~s}^{-1}\right)$ and (b) 5-min change in TVS $\Delta V$ (m s$\left.{ }^{-1}\right)$ at the radar level closest to $500 \mathrm{~m}$ ARL for the final volumes TVSs were identified in (labeled "D") and the three volumes prior (D-1, D-2, D-3). The box-and-whisker plots show median and 25th and 75th percentiles within the box and minimum and maximum values as whiskers unless the values were outliers, in which case the outlier is plotted beyond the whiskers. If the number of cases for each volume was not constant, then the sample size appears beneath the volume in question. If the volume label for D-1, D-2, or D-3 appears as red, then the null hypothesis that the difference between the population mean ranks of that volume and volume $\mathrm{D}$ are zero can be rejected at the $1 \%$ level ( $p$ value $\leq 0.01)$ using a directional Wilcoxon signed-rank test.

contributor toward more rigorously identifying future metric possibilities for use in nowcasting applications.

\section{a. TVS intensity}

The maximum $\Delta V$ value within the TVS was recorded at the radar center beam height (ignoring atmospheric effects and elevation changes) closest to $500 \mathrm{~m}, 750 \mathrm{~m}, 1 \mathrm{~km}, 2 \mathrm{~km}$, and $3 \mathrm{~km}$ for each volume that met the requirements set out in section 2. In some cases when the range from the radar to the TVS in question was large, the scan closest in height to $500 \mathrm{~m}(750 \mathrm{~m})$ also was the scan closest in height to $750 \mathrm{~m}(1 \mathrm{~km})$, and this may contribute to similarities between TVS behaviors at different height levels.

At $500 \mathrm{~m}$, TVS intensity is noticeably lower in the last observed volume with a TVS (hereafter "D volume") than in the previous three volumes (Fig. 4a). TVS intensity in the three volumes prior to the $\mathrm{D}$ volume are largely similar with median values $50-60 \mathrm{~m} \mathrm{~s}^{-1}$, but in the last volume, the median value is much lower, at $\sim 32 \mathrm{~m} \mathrm{~s}^{-1}$, and the $25 \mathrm{th}-75$ th-percentile values do not overlap with those in the three prior volumes. In addition, the range and variance of the values decrease approaching dissipation. The larger intensity spreads in the D-3 and D-2 volumes likely result from these volumes incorporating cases representing different parts of the tornado life cycle, whereas in the D volume all of the 36 cases represent tornadoes in their dissipation phases. The change in TVS intensity in successive volumes normalized to $5 \mathrm{~min}$ (Fig. 4b) is largest for the final two volumes, both of which display median decreases in intensity. However, the decrease is larger and more consistent in the 5-min period between the last two volumes; in 33 of the 36 dissipation cases, there is a decrease in intensity leading up to the $\mathrm{D}$ volume. However, intensity decreases also are common well prior to dissipation, in the D-3 volume. The differences in intensity and intensity change between the $\mathrm{D}$ volume and the three prior volumes are all significant at the $1 \%$ level. An example of a weakening TVS consistent with these results is shown in Fig. 5; the TVS intensity was relatively steady initially (Figs. 5a-c) before decreasing rapidly in its final $10 \mathrm{~min}$ (Figs. 5d,e) and dissipating (Fig. 5f).

The same pattern of weaker and weakening TVSs in the D volume follows at other height levels (e.g., Fig. 6). TVS intensity and intensity change using data from the radar level closest to $750 \mathrm{~m}$ (not shown) are nearly identical to those from $500 \mathrm{~m}$. Between 1 and $2 \mathrm{~km}$ (Figs. 6a,b), however, there is a large drop in median and 25th and 75th-percentile TVS intensities in the D-1 volume (red boxes) that is not seen in the other volumes. 


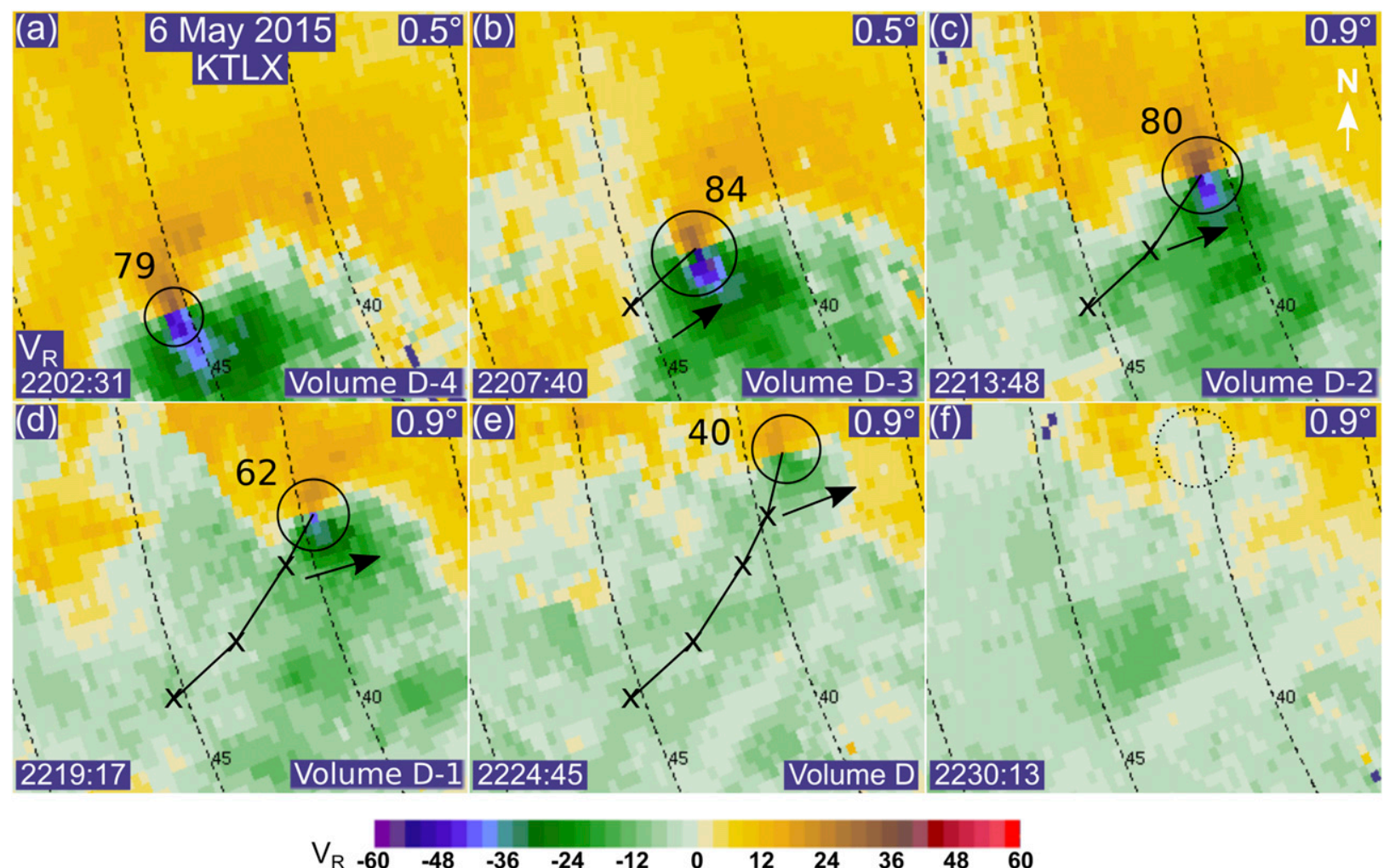

FIG. 5. Radial velocity $\left(\mathrm{m} \mathrm{s}^{-1}\right)$ from the WSR-88D in Twin Lakes, OK (KTLX), of a dissipating TVS associated with a tornado on 6 May 2015 at (a) 2202:31, (b) 2207:40, (c) 2213:48, (d) 2219:17, (e) 2224:45, and (f) 2230:13 UTC. The black circles outline the TVS and the number next to the outline is the estimate of TVS intensity via the maximum TVS $\Delta V\left(\mathrm{~m} \mathrm{~s}^{-1}\right)$. The $\times$ annotations mark the approximate volume-by-volume progression of the TVS over its last $\sim 25 \mathrm{~min}$ and the arrows indicate the estimated storm motion direction for that volume. Range rings are every $5 \mathrm{~km}$. The images are all centered at the same location. The increase in elevation angle from (b) to (c) results from the TVS moving toward the radar, which necessitates a higher angle to get closest to a center beam height of $\sim 500 \mathrm{~m}$ ARL; all times have TVS heights of $\sim 400-600 \mathrm{~m}$ ARL. This case is separate from the 6 May 2015 case shown in Fig. 2.

In addition, there is more 5-min weakening entering the $\mathrm{D}$ volume (median decrease of $\sim 20 \mathrm{~m} \mathrm{~s}^{-1}$ ) at $1 \mathrm{~km}$ (Fig. 6c) than at the other height levels (medians of $\sim 10-15 \mathrm{~m} \mathrm{~s}^{-1}$; e.g., Fig. 6d). One speculative explanation for both of these observations is that TVS dissipation tends to occur first in the 1-2-km layer, and then progresses upward and downward from that level, as observed in three cases by French et al. (2014) and in one case by Houser et al. (2015). If there were such a progression in time-height dissipation, then one would expect weaker TVSs and a maximum in weakening first at $2 \mathrm{~km}$ in the D-1 volume, followed by weakening at $500 \mathrm{~m}$ and $1 \mathrm{~km}$ in the D volume, which is consistent with the results shown in Figs. 4 and 6. However, accurate case-by-case analysis of timeheight dissipation requires radar volumetric update times much greater than that afforded by WSR-88D systems.

\section{b. TVS storm-relative motion}

Using the $\sim 15$-min average storm motion, the SR movements of the TVSs were estimated in their final four volumes at several height levels. The previous work motivating this part of the study universally examines tornado motion in a forward-rearward, leftwardrightward framework relative to the storm (see section 1) rather than using cardinal directions. Therefore, SR TVS motions were transformed into forward-rearward and rightward-leftward components. At $500 \mathrm{~m}$ (Fig. 7), the magnitudes of the SR TVS motions increase as TVSs approach dissipation. In the D-3 (Fig. 7a) and D-2 (Fig. 7b) volumes, TVS motions are similar to storm motions (SR motions $<5 \mathrm{~m} \mathrm{~s}^{-1}$ magnitude) and variable in direction. In the final two TVS volumes (Figs. 7c,d), there are larger SR components to motion, and the SR directions are consistently, but not exclusively, rearward.

To better determine the relationship between cyclic tornadogenesis and SR motion, the cases were individually inspected to identify whether cyclic tornadogenesis was occurring. Any case in which the tornado under question was preceded (followed) by a tornado within $20 \mathrm{~min}$ of the genesis (dissipation) time was categorized as a cyclic tornadogenesis (hereafter "cyclic") case. A case was additionally categorized as cyclic if 


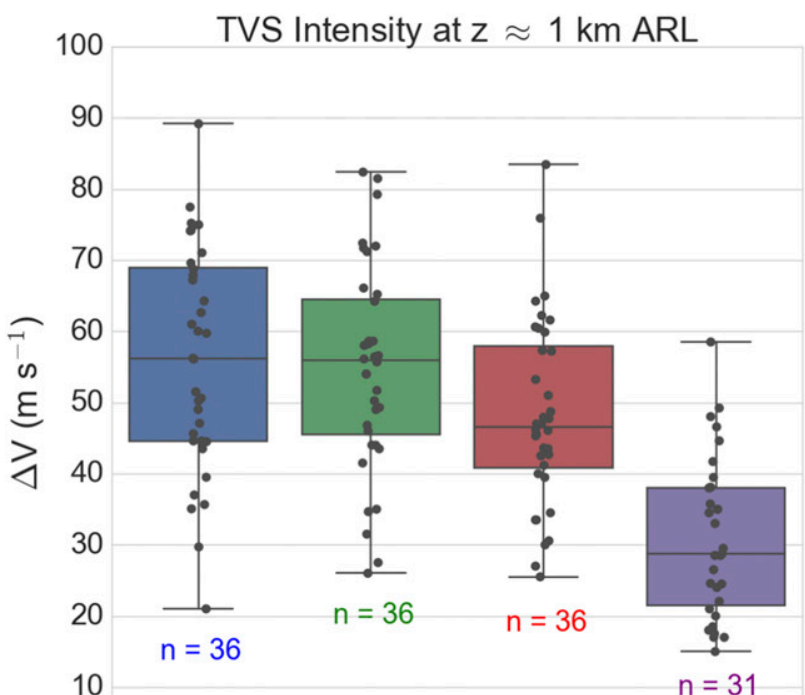

(a)

${ }_{0}^{(a)}$

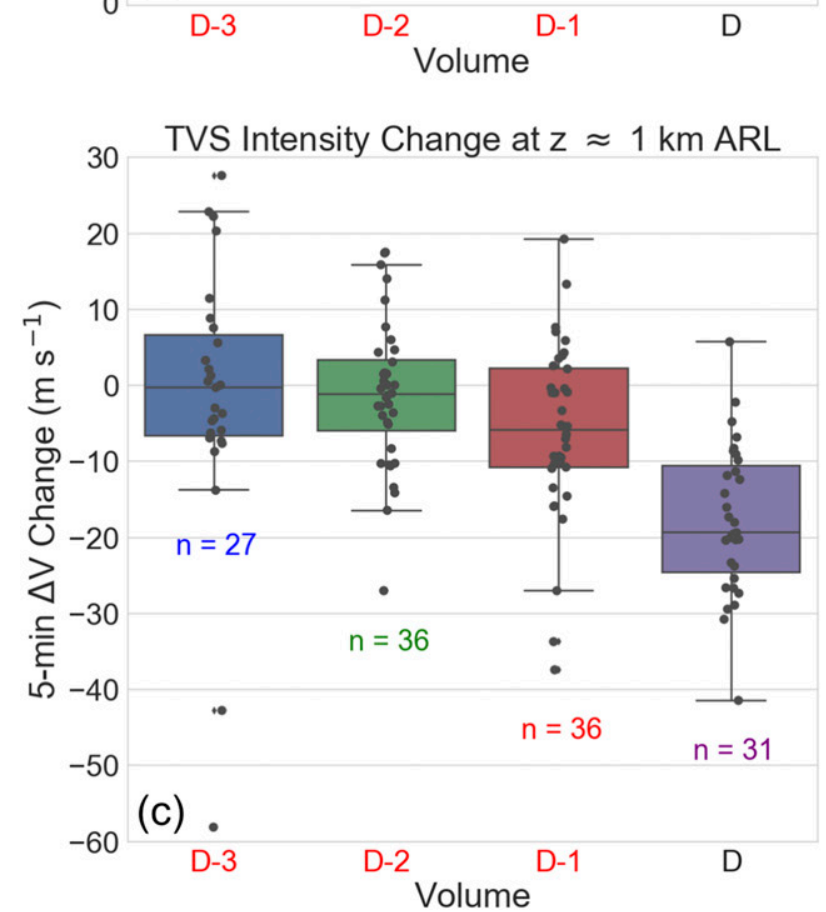

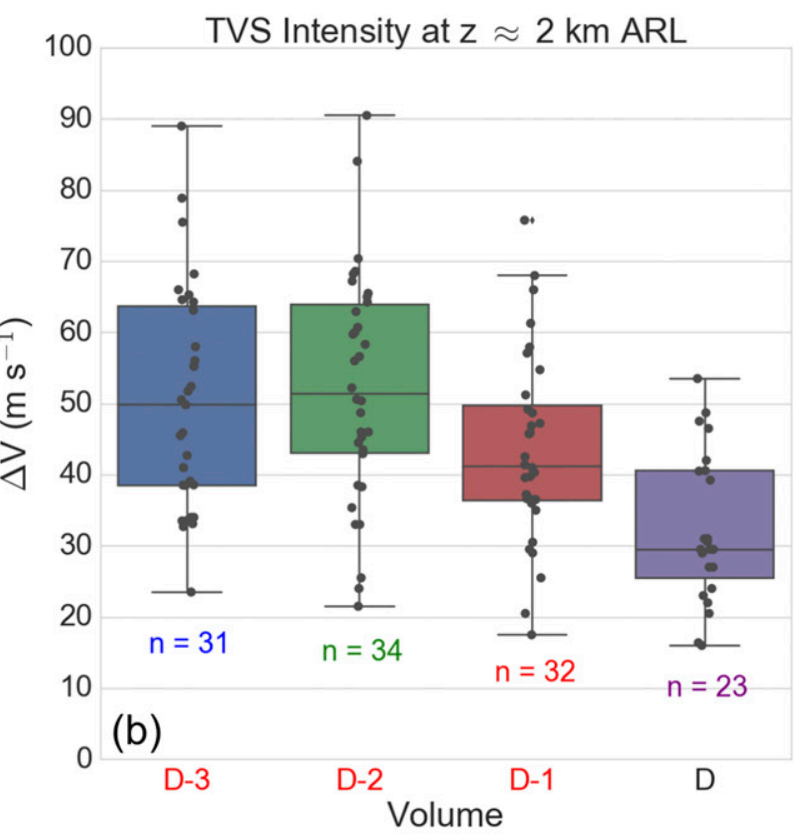

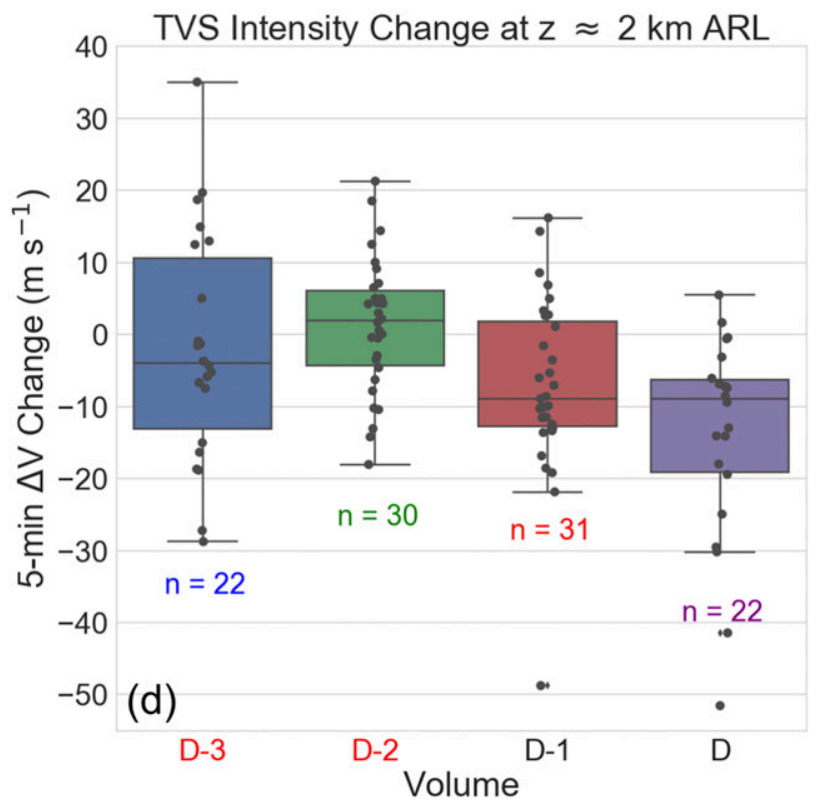

FIG. 6. (a),(b) As in Fig. 4a, but using data from the radar level closest to 1 and 2 km ARL, respectively; (c),(d) as in Fig. 4b, but using data from the radar level closest to 1- and 2-km ARL, respectively. Note the different 5-min $\Delta V$ change intervals used in (c) and (d).

there was a preceding or subsequent strong low-level vortex signature but no confirmed tornado (i.e., if cyclic mesocyclogenesis, but not confirmed cyclic tornadogenesis, was occurring). In Fig. 7d, the cases are separated out as cyclic (violet) and noncyclic (cyan): there is a strong tendency for TVSs within (outside of) the cyclic tornadogenesis or mesocyclogenesis process to dissipate as they move leftward (rightward) relative to the motion of the storm.
To visualize differences among the individual motion components, box-and-whisker plots also were constructed (Fig. 8). The increase in the magnitudes of TVS SR motions (Fig. 8a), and tendency toward rearward motions (Fig. 8b) and leftward or rightward motions (Fig. 8c) as TVS dissipation nears can be seen as a progressive widening of the distributions with time. However, there is large overlap among all the distributions and there is minimal statistically significant difference 

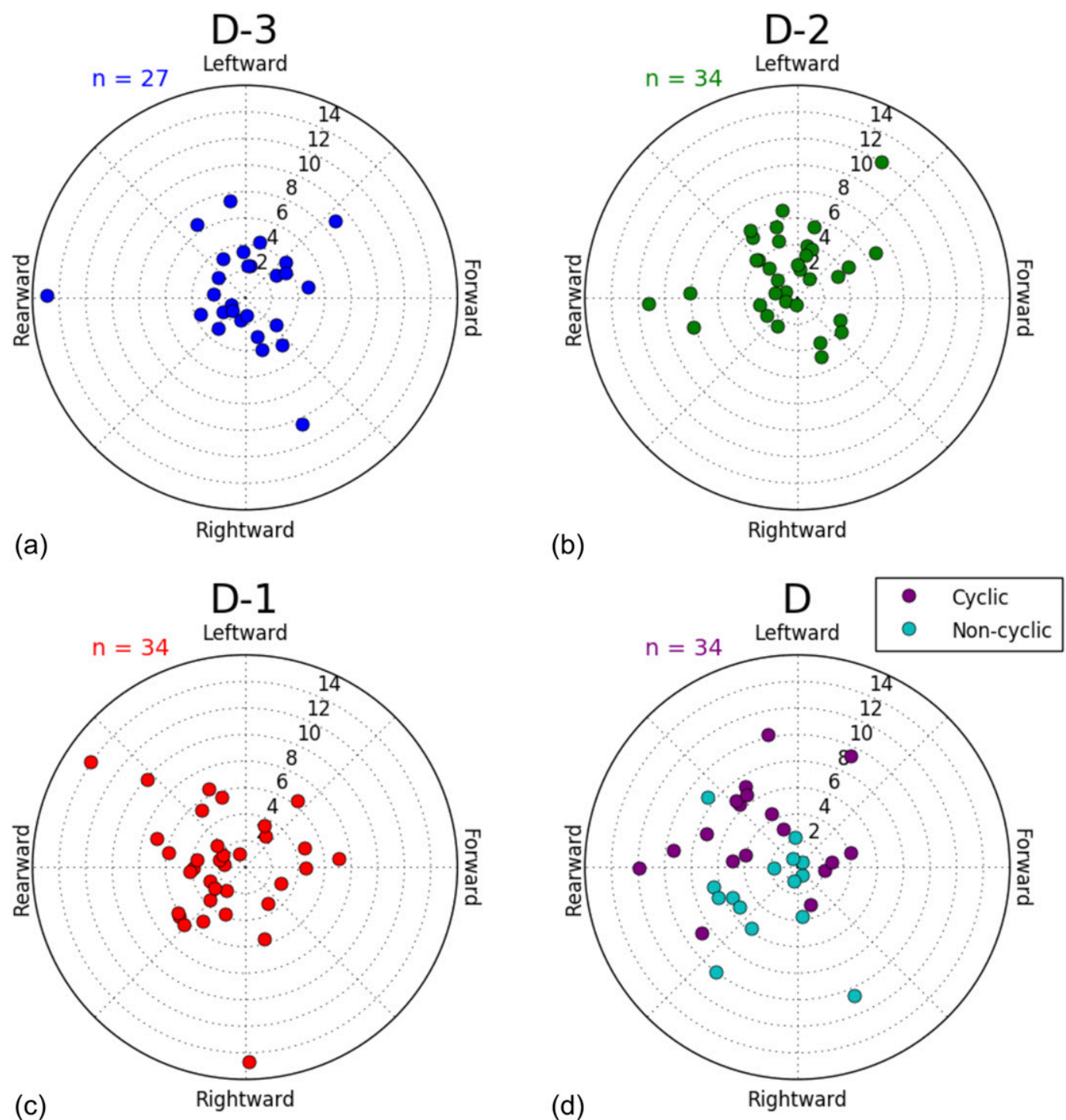

FIG. 7. Polar scatterplots of storm-relative TVS motion at the radar level closest to $500 \mathrm{~m}$ ARL from the (a) D-3, (b) D-2, (c) D-1, and (d) D volume, as described previously. The range from the origin denotes the storm-relativemotion magnitude $\left(\mathrm{m} \mathrm{s}^{-1}\right)$, and the angles represent the direction of storm motion in forward-rearward and rightward-leftward components, as labeled in each panel. In (d), the cases are further parsed between storms that exhibited cyclic tornadogenesis or cyclic mesocyclogenesis and those that did not. For visual convenience, one outlier case (magnitude $\sim 20 \mathrm{~m} \mathrm{~s}^{-1}$ ) is omitted from (d).

between the $\mathrm{D}$ volume motions and those from the three prior volumes. There is a much clearer separation of the rightward-leftward component motion distributions between cyclic and noncyclic cases in the final two volumes (Fig. 8d), although sample sizes are low as the dataset was additionally parsed, and statistical tests are likely to be unreliable. An example of a TVS at $500 \mathrm{~m}$ exhibiting the summarized dissipation behaviors within the cyclic tornadogenesis process is shown in Fig. 5. The TVS initially moved in a similar direction as the storm (Figs. 5a,b), but then curved to the left (north) and slowed (Figs. $5 \mathrm{c}-\mathrm{e}$ ) relative to the storm before dissipating (Fig. 5f).

The progression of changes in SR motions also was investigated to determine if SR motions become increasingly more rearward for dissipating TVSs and increasingly more leftward (rightward) for cases exhibiting (not exhibiting) cyclic tornadogenesis or mesocyclogenesis (not shown). Most 5-min changes in the TVS SR forward-rearward components are $<5 \mathrm{~m} \mathrm{~s}^{-1}$ and the maximum rearward TVS motion tendencies occur between volumes D-2 and D-1. Similarly, virtually all 


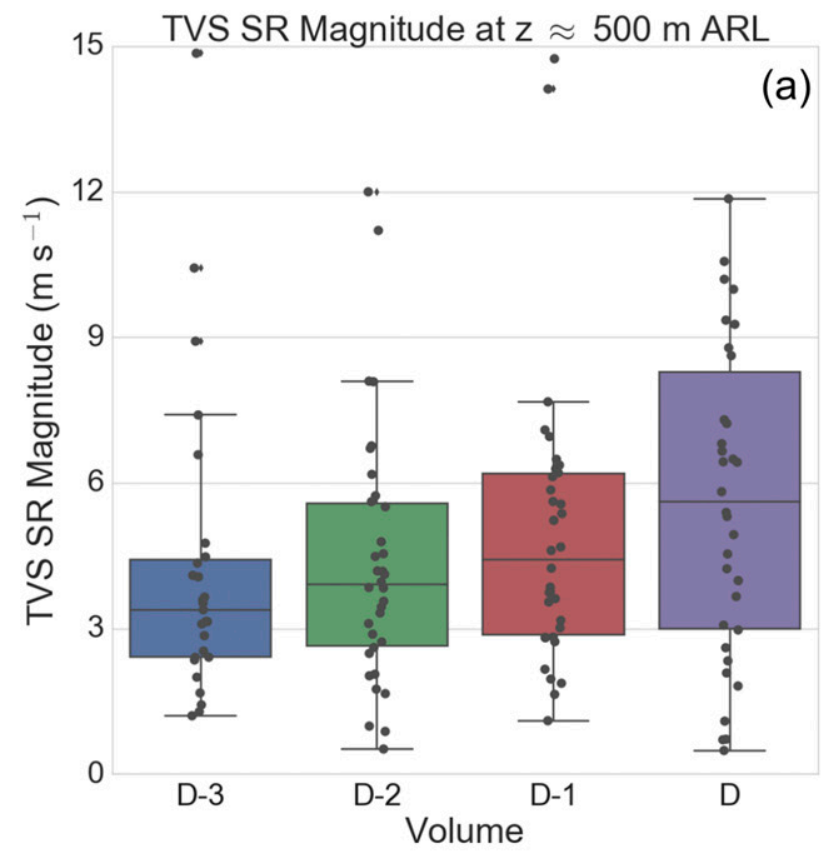

10 TVS SR For-Rear Motion at $z \approx 500 \mathrm{~m} \mathrm{ARL}$

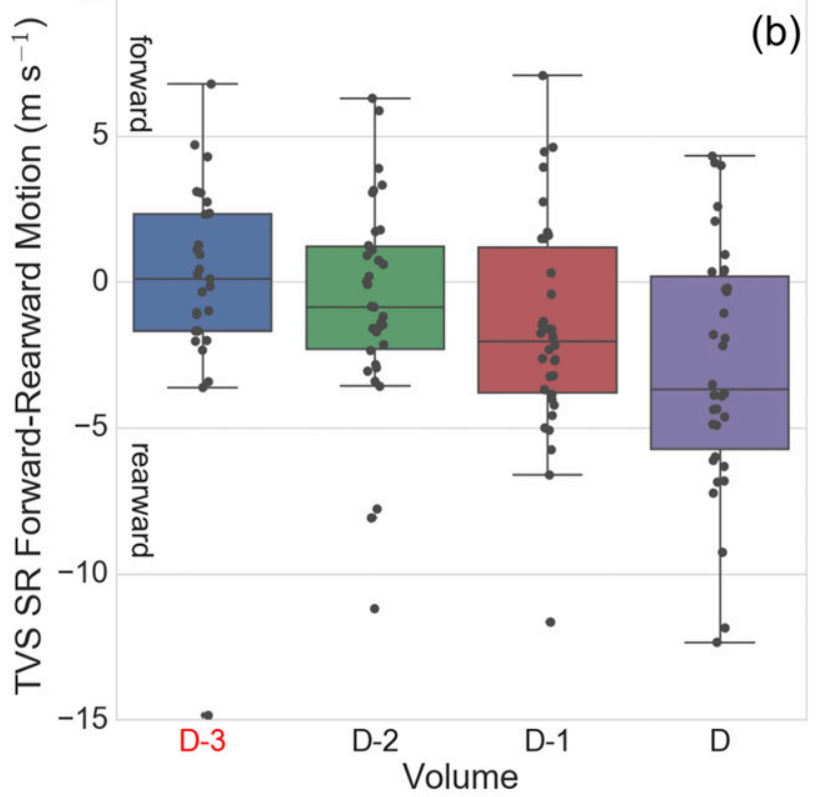

TVS SR L/R Magnitude at $z \approx 500 \mathrm{~m} \mathrm{ARL}$
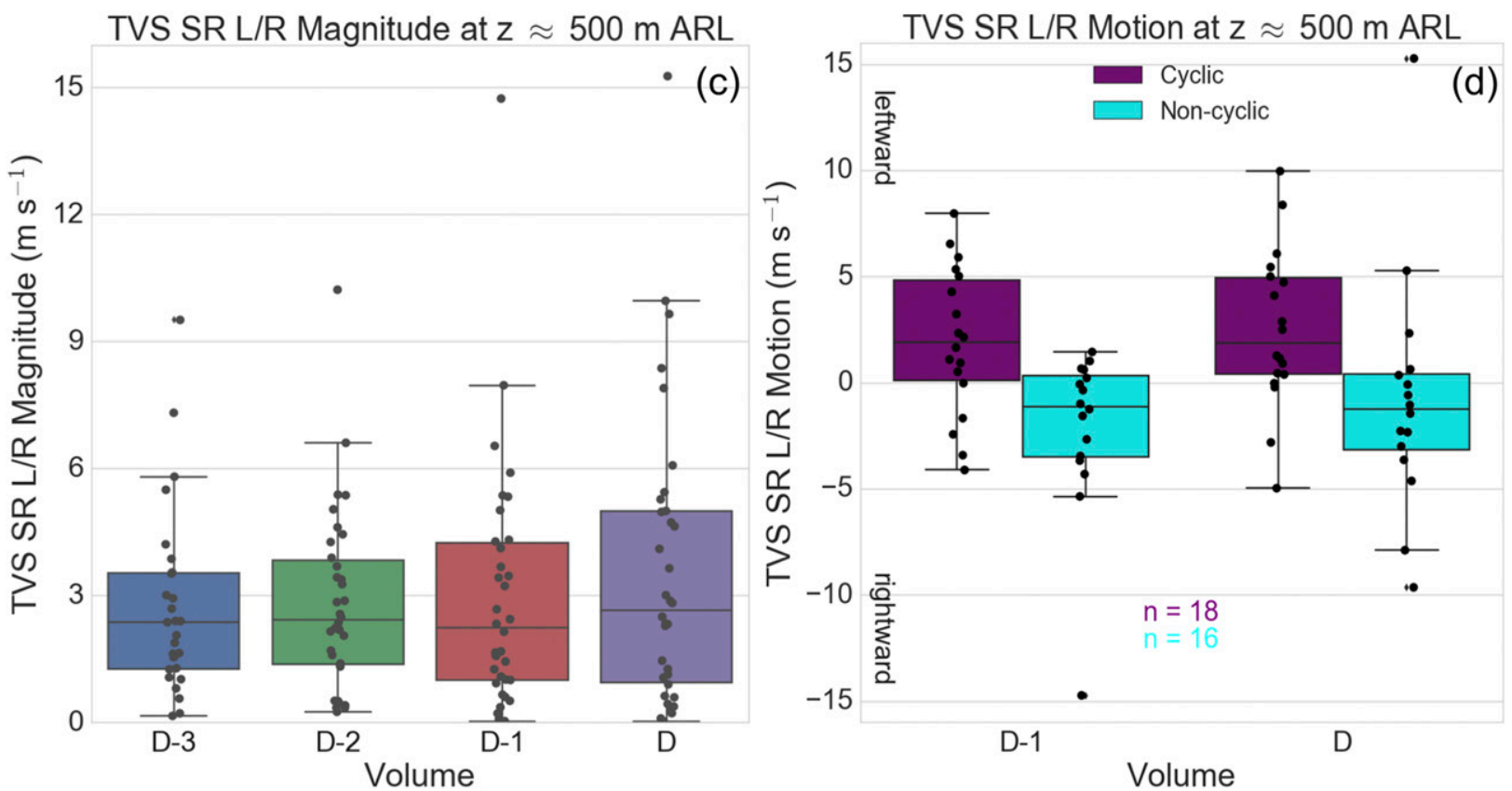

FIG. 8. As in Fig. 4, but for TVS storm-relative (a) total motion magnitude, (b) forward-rearward component motion, (c) leftwardrightward component magnitude, and (d) leftward-rightward component motion. In (d), only D-1 and D volumes are shown and the cases are parsed by the presence of cyclic tornadogenesis or mesocyclogenesis. Statistical testing was not performed on the categorical data shown in (d) owing to small sample sizes. Samples sizes in (a)-(c) are the same as those shown in Fig. 6.

SR rightward-leftward tendencies are $<4 \mathrm{~m} \mathrm{~s}^{-1}$ in volumes D-1 and D and there is substantial overlap in the distributions between cyclic and noncyclic storms. There is no statistically significant difference among volumes for the tendency measurements.

Finally, SR motions were examined at additional height levels (e.g., Fig. 9). The progressions of TVS SR motions among the final four volumes for the cases are qualitatively similar at the 500-m and $1-\mathrm{km}$ levels, including larger SR magnitudes and SR rearward motions in volume D (Fig. 9d) than in the earlier three volumes (Figs. 9a-c). However, the magnitudes of both of these trends are less than those seen at $500 \mathrm{~m}$, which is evidence that TVS motions are more similar to that of the 

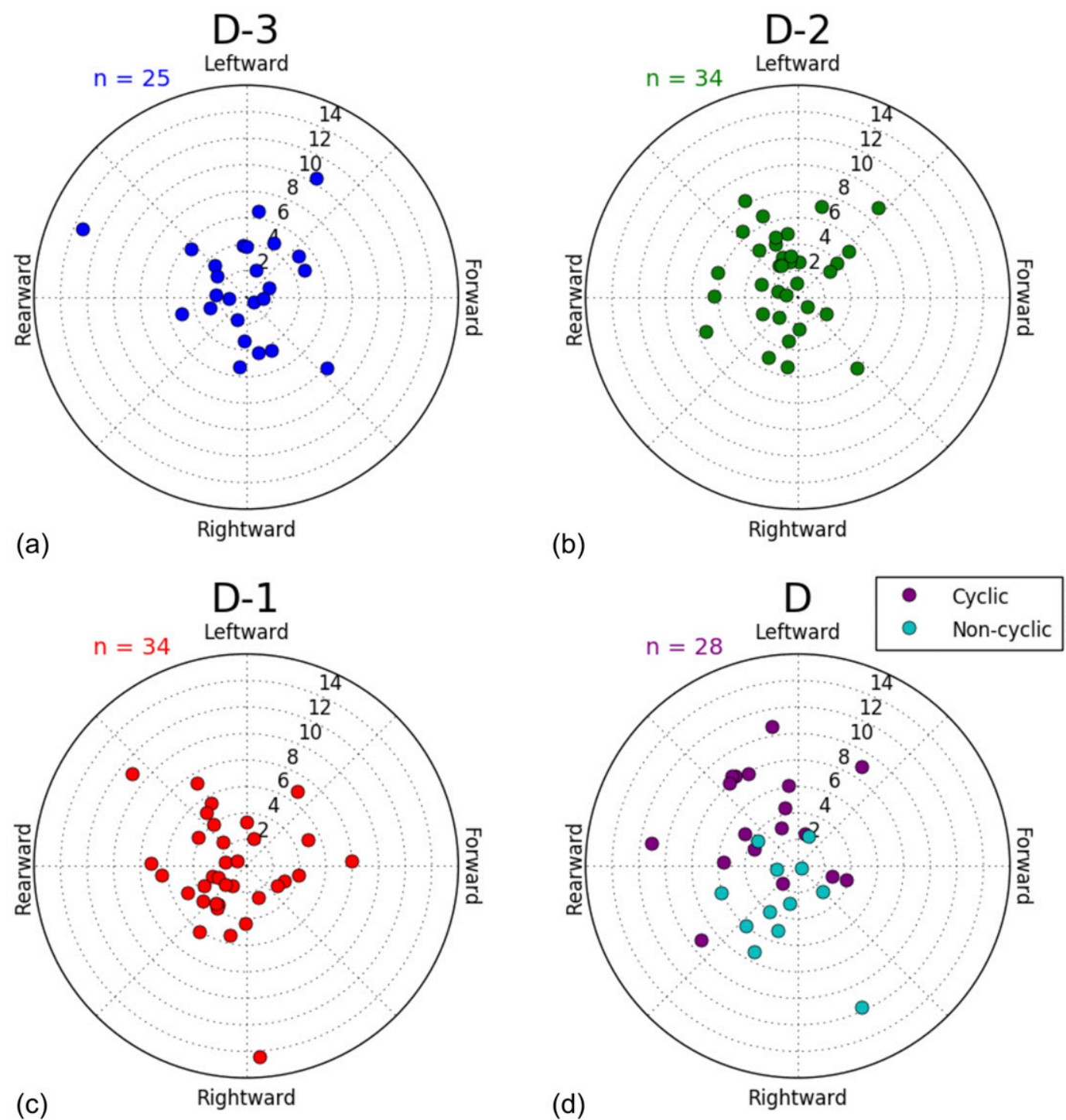

FIG. 9. As in Fig. 7, but at the radar level closest to $1 \mathrm{~km}$ ARL.

storms above the lowest 500-m level than at or below the 500-m level. SR magnitudes at $2 \mathrm{~km}$ (not shown) are similar to those at $1 \mathrm{~km}$. At $1 \mathrm{~km}$, the separation between cyclic and noncyclic cases in rightward-leftward component motions in volumes D-1 and D is similar to that seen at $500 \mathrm{~m}$ (Fig. 9d); the separation also is evident at $2 \mathrm{~km}$ (not shown), but the number of cases in the D volume is only 21 .

\section{c. TVS tilt}

For a subset of the 36 cases, those in which a TVS was identified at 2- and/or 3-km heights, TVS tilt was estimated using the location of the TVS at two levels. The tilt is expressed as the inclination angle so that differences in the exact heights of the levels were accounted for in the calculations. In addition, the time between scans introduces spatial differences that must be accounted for by advection correction. To do so, the TVS motion at the higher level (i.e., at 2 or $3 \mathrm{~km}$ ) between the previous volume and current volume was used to estimate the spatial offset that accrued in the time between the 500-m and 2- or 3-km observations within the same volume.

The inclination angles between the scans closest to $500 \mathrm{~m}$ and those closest to $2 \mathrm{~km}$ (Fig. 10a) and $3 \mathrm{~km}$ (Fig. 10b) do not have obvious trends with time during the end of TVS life cycles. In general, inclination angles are highly variable, with most cases commonly exhibiting values of $15^{\circ}-45^{\circ}$. For both $0.5-2.0-$ and $0.5-3.0-\mathrm{km}$ TVS tilt, the D volume has the highest median inclination angle, but it is not statistically different from 

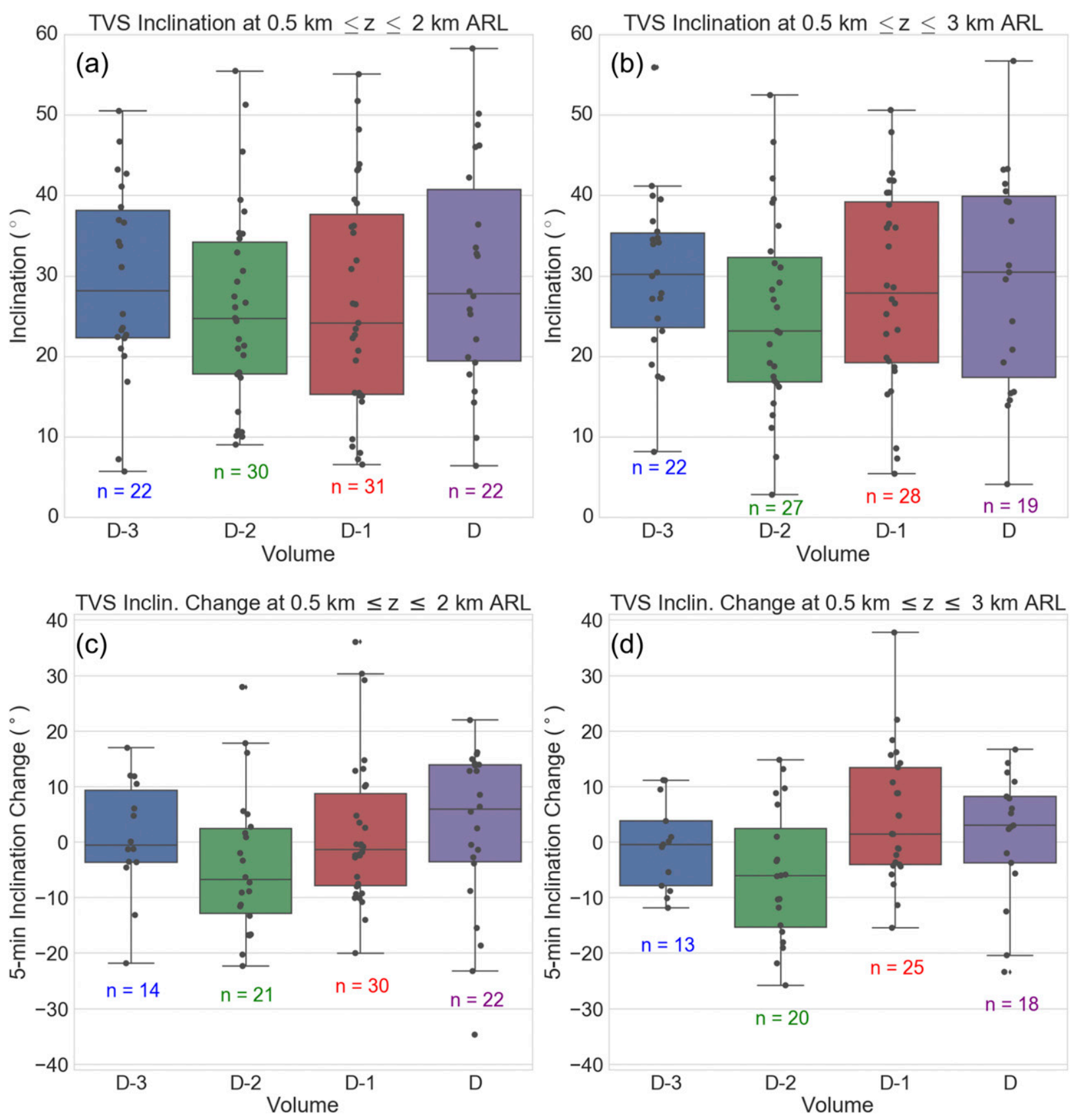

FIG. 10. (a),(b) As in Fig. 4a, but for TVS inclination angle from $\sim 0.5$ to $2 \mathrm{~km}$ and from $\sim 0.5$ to $3 \mathrm{~km}$, respectively; (c), (d) as in Fig. $4 \mathrm{~b}$, but for TVS inclination angle from $\sim 0.5$ to $2 \mathrm{~km}$ and $\sim 0.5$ to $3 \mathrm{~km}$, respectively.

earlier volumes. The 5-min changes in TVS tilts also were examined between the $500-\mathrm{m}$ level and the $2-\mathrm{km}$ (Fig. 10c) and 3-km (Fig. 10d) levels. The qualitative progression as dissipation approaches is the same for both parameters: a tendency toward more vertical TVSs from D-3 to D-2, followed by increases in TVS tilts through dissipation. Most observations (17/24 cases) of large increases in inclination angle $\left[>10^{\circ}(5 \mathrm{~min})^{-1}\right]$ occurred in the D-1 and D volumes for $0.5-2.0-\mathrm{km}$ tilt.
The paired data included sample sizes too low $(n<15)$ to reliably test for statistical significance.

\section{d. TVS displacement from the midlevel updraft}

The final TVS behavior analyzed is how much horizontal distance the TVS accrues away from the main storm (i.e., midlevel) updraft. The horizontal location of the midlevel updraft was approximated by using the same centroid of the $Z_{\mathrm{DR}}$ column as that used in estimating 

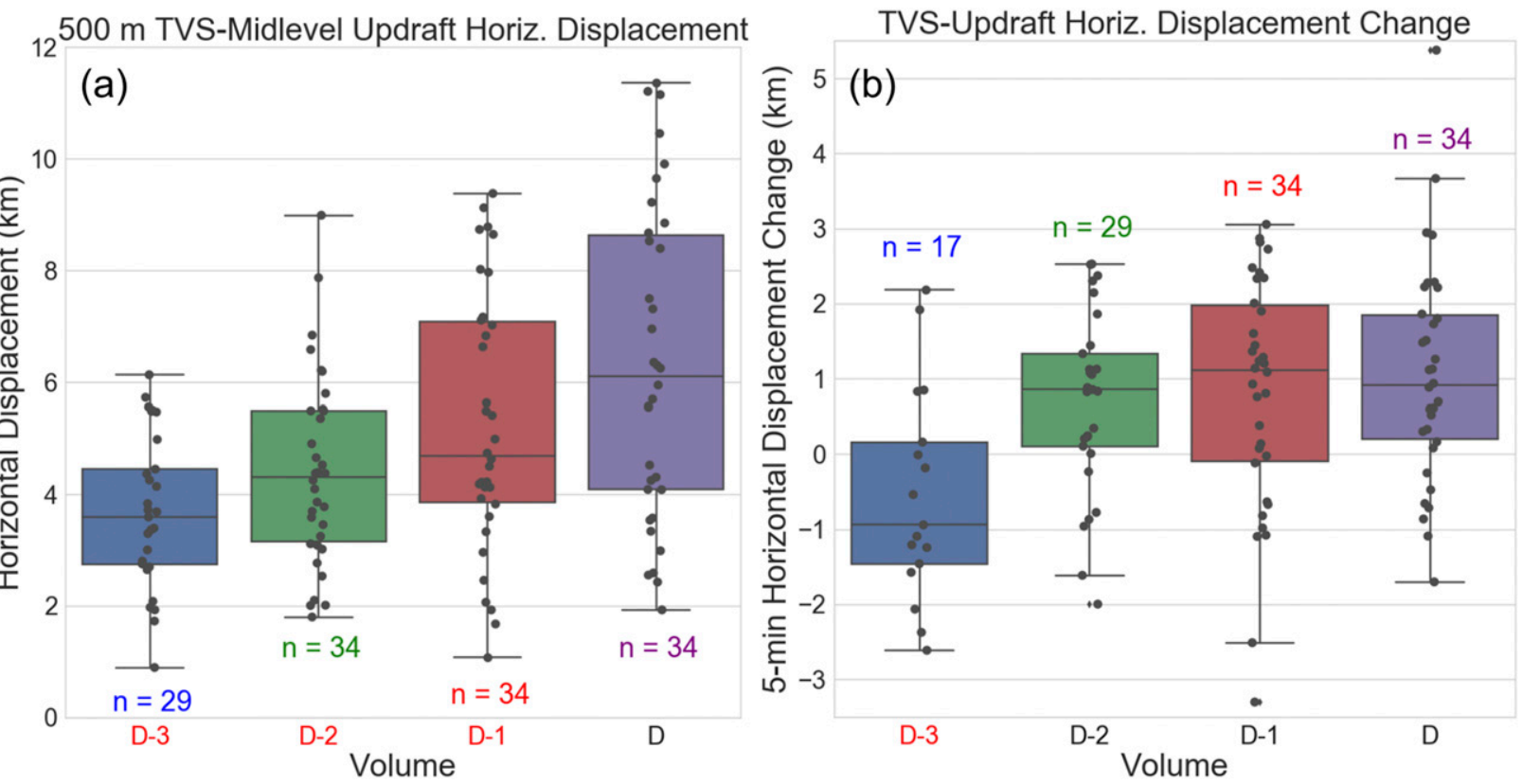

FIG. 11. As in Fig. 4, but for the horizontal displacement between the TVS at $500 \mathrm{~m}$ and the approximate location of the midlevel storm updraft.

storm motion (e.g., Fig. 2). The displacement calculation used the location of the features (TVS at $500 \mathrm{~m}$ and $Z_{\text {DR }}$ column centroid at $\sim 4-7 \mathrm{~km}$ ) at two different height levels within the same radar volume, so a similar advection correction process described in section $3 \mathrm{c}$ was used to correct for the spatial offset between the two levels, except that storm motion was used instead of TVS motion. One limitation to this approach is that the

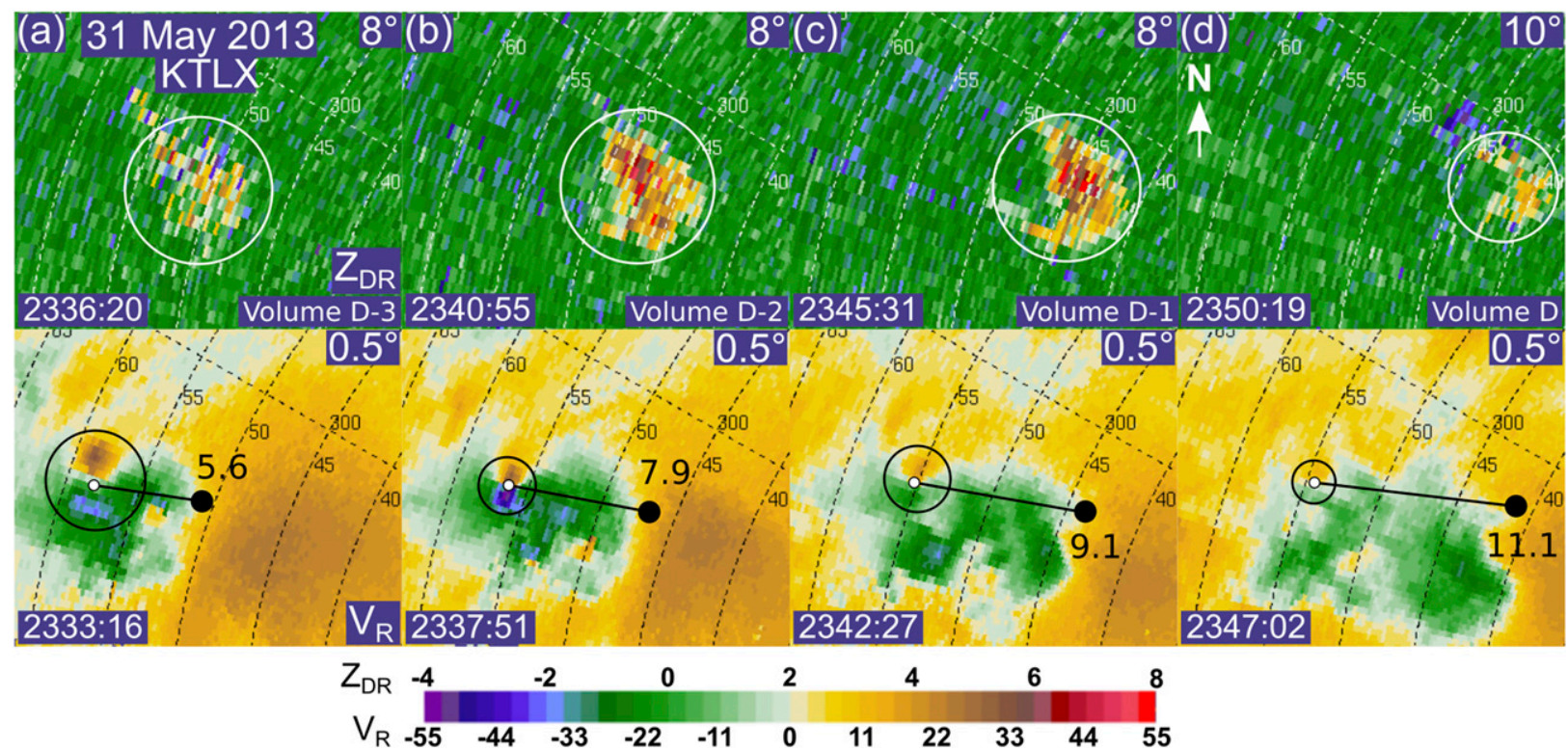

FIG. 12. (top) Differential radar reflectivity (dB) and (bottom) radial velocity ( $\mathrm{m} \mathrm{s}^{-1}$ ) from the WSR-88D in Twin Lakes, OK (KTLX), of a dissipating TVS associated with a tornado on 31 May 2013 at volumes beginning at approximately (a) 2333, (b) 2338, (c) 2342, and (d) 2347 UTC. The white circle outlines the approximate location of the $Z_{\mathrm{DR}}$ column associated with the storm updraft. The black circle outlines the TVS, and the black line connects the approximate centers of the TVS (filled white circle) to the reflection of the centroid of the $Z_{\mathrm{DR}}$ column onto the lower surface (filled black circle). The number next to the black line is the horizontal distance (km) between the center of the TVS and the centroid of the $Z_{\mathrm{DR}}$ column. The distance values shown incorporate advection correction that the images cannot account for. The images are all centered at the same location. The heights of the center of the radar beam at the locations of the $Z_{\mathrm{DR}}$ column (TVS) vary from $\sim 6$ to $7 \mathrm{~km}(\sim 400$ to $600 \mathrm{~m})$ ARL. 
height levels of the updraft observations are variable, owing to the 34 cases having different optimal levels for $Z_{\mathrm{DR}}$ column identification. Most observations were made at $4.5-6.0 \mathrm{~km}$ in height, so a heavily tilted $Z_{\mathrm{DR}}$ column may bias horizontal displacement values. In practice, there was little observed horizontal variability in column centroids at midlevels when the column was identified at multiple levels.

TVSs in their final volume tend to be located farther from the midlevel storm updraft than in any of the previous volumes (Fig. 11a). Median distances increase a small amount from $\sim 3.5$ to $\sim 4.75 \mathrm{~km}$ in the D-3 to D-1 volumes and then there is a larger increase to over $6 \mathrm{~km}$ in the $\mathrm{D}$ volume. The variability of horizontal displacement distances also increases approaching TVS dissipation, and the distribution is noticeably broader in the D-1 and $\mathrm{D}$ volumes compared to the previous two volumes. The 5 -min changes in the spatial location offsets also were computed (Fig. 11b). Earlier cases (D-3 and D-2 volumes) are split between increasing and decreasing TVSupdraft displacements, but in the later cases (D-1 and D volumes), a large majority $(51 / 68 ; 75 \%)$ of observations are of TVSs becoming displaced farther horizontally from the approximate location of the midlevel updraft. An example of this behavior is shown in Fig. 12, in which the TVS was initially within $\sim 5.6 \mathrm{~km}$ horizontally of the midlevel updraft (Fig. 12a) but slowed relative to the storm and became displaced gradually farther away from it in each of the final TVS volumes (Figs. 12b-d).

\section{e. TVS dissipation behavior relationships and combinations}

The four TVS dissipation behaviors and their trends may correlate with each other. A large SR TVS magnitude could lead to a large TVS horizontal displacement away from the main storm updraft if the motion is consistent directionally (e.g., large rearward motion followed by large forward motion would result in small horizontal displacement). Likewise, a large SR TVS magnitude could lead to an increase in TVS tilt if the motion of the TVS aloft is similar to that of the storm. If behaviors are well correlated, then they may be redundant in what they convey about the supercell and tornado, and the need to use them separately in operations is lessened.

Scatterplots were constructed of SR TVS motion versus TVS tilt and horizontal displacement (Fig. 13a) and 5-min changes in the forward-rearward SR component versus 5 -min changes in $0.5-2.0-\mathrm{km}$ tilt and horizontal displacement (Fig. 13b). None of the four scatterplots demonstrates evidence of strongly correlated behaviors, and no Pearson correlations are larger in absolute value than 0.38 . Additional scatterplots
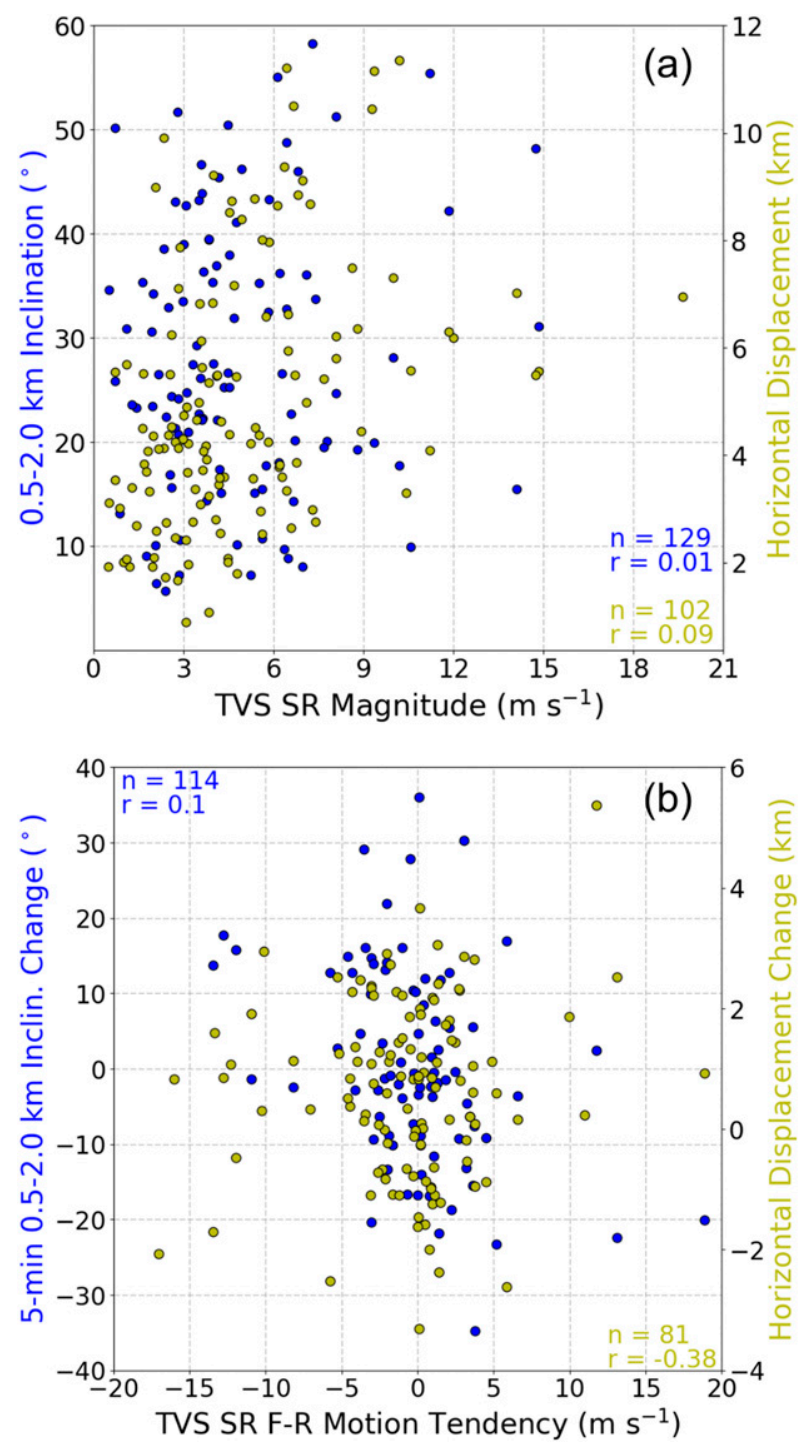

FIG. 13. Scatterplots of (a) TVS SR magnitude vs $0.5-2.0-\mathrm{km}$ inclination (blue) and vs horizontal displacement between the TVS at $500 \mathrm{~m}$ and the approximate location of the midlevel storm updraft (yellow) and (b) 5-min change in TVS forward-rearward component vs 5 -min change in $0.5-2.0-\mathrm{km}$ inclination (blue) and vs 5 -min change in horizontal displacement between the TVS at $500 \mathrm{~m}$ and the approximate location of the midlevel storm updraft (yellow). Color-coded sample sizes $n$ and Pearson correlation coefficients $r$ also are included.

between $0.5-2.0-\mathrm{km}$ TVS tilt and TVS-updraft horizontal displacement and their trends also show almost no linear correlation between the parameters (not shown). The lack of a strong linear relationship between SR motion and tilt is consistent with the wide range of TVS inclination angles observed; in some cases large near-surface rearward SR motion was paired with similar behavior aloft (small inclination angle) and in other cases SR motion differed substantially in the vertical 
(a)

D-1

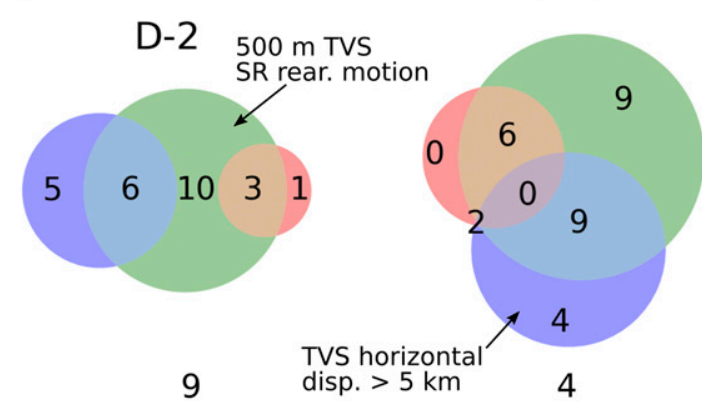

(b)

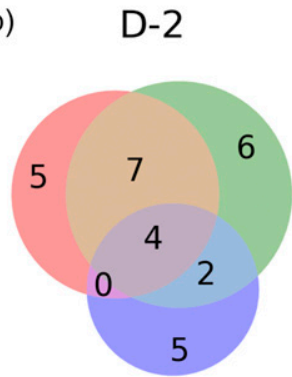

5

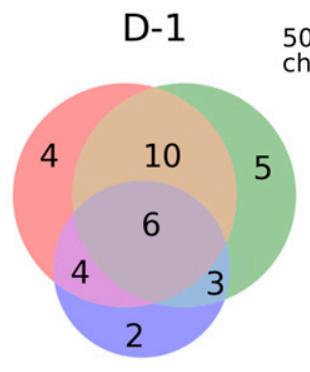

0

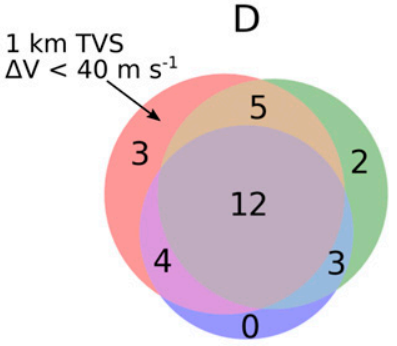

1

FIG. 14. Area-proportional Venn diagrams of TVS dissipation behavior combinations in the three final volumes of TVSs. (a) Rearward SR TVS motion at $500 \mathrm{~m}$ ARL (green), TVS horizontal displacement $>5.0 \mathrm{~km}$ (purple), and 1-km TVS $\Delta V<40 \mathrm{~m} \mathrm{~s}^{-1}$ (red). (b) As in (a), but, for intensity, 5-min 500-m TVS $\Delta V$ change $<0 \mathrm{~m} \mathrm{~s}^{-1}$ (red). Only cases in which all three behaviors could be determined were included, which numbered 34 for (a) and (b), except for volume D in (a), which had 30 cases. Two (three) simultaneous behaviors are denoted by brown, blue, and/or magenta (muted purple). The numbers immediately below the diagrams are the null set for the 30 or 34 cases.

(large inclination angle). The absence of a linear relationship between SR motion and horizontal displacement is consistent with small TVS SR magnitudes and variable directions prior to the $\mathrm{D}$ volume.

Similarly, it is possible that the presence of multiple behaviors may be more likely at the time of dissipation than at earlier times (e.g., Fig. 5). To examine these combinations, Venn diagrams using TVS intensity, SR motion, and horizontal displacement parameters were constructed (Fig. 14) for the final three volumes (the D-3 volume sample sizes were too low to assess). For example, one set of clear dissipation behaviors for each category is TVS $\Delta V<40 \mathrm{~m} \mathrm{~s}^{-1}$ at $1 \mathrm{~km}, 500-\mathrm{m}$ SR rearward component motion, and 500-m TVS displacement from the midlevel updraft $>5 \mathrm{~km}$ (Fig. 14a). Combinations of behaviors become more common approaching dissipation [24/30 cases (80\%) at D vs 9/34 cases (26\%) at D-2], including all three behaviors occurring simultaneously [12/30 cases $(40 \%)$ at $\mathrm{D}$ vs $0 / 34$ cases $(0 \%)$ at $\mathrm{D}-2]$. However, one potential problem with using TVS intensity cutoffs as a predictor of tornado dissipation is the need in this study to analyze only long-duration tornadoes, which are likely stronger in intensity than the set of all tornadoes (e.g., Fig. 3). Therefore, also shown is TVS 5-min $\Delta V$ decrease (weakening) at $500 \mathrm{~m}$ (Fig. 14b) instead of TVS intensity at $1 \mathrm{~km}$; the other two behaviors are unchanged. Again, all three behaviors occurring for a case becomes much more likely in the D volume (16/34; $47 \%)$ compared to the D-2 volume (4/34; $12 \%)$.

Despite the prevalence of combinations of dissipation behaviors in the D-1 and D volumes, all of the summarized behaviors 1) are common at other times earlier in tornado life cycles, including combinations of these behaviors (e.g., Fig. 15a), and 2) are occasionally absent from the final TVS volumes (e.g., Fig. 15b). In the former example, TVS intensity decreased and SR motion was rearward, but the TVS persisted for another $15+$ min such that either there was an extended dissipation process or the behaviors were not ultimately a reflection of a dissipating tornado. In the latter example, the TVS intensity increased and moved with almost no SR motion despite entering its final volume. 


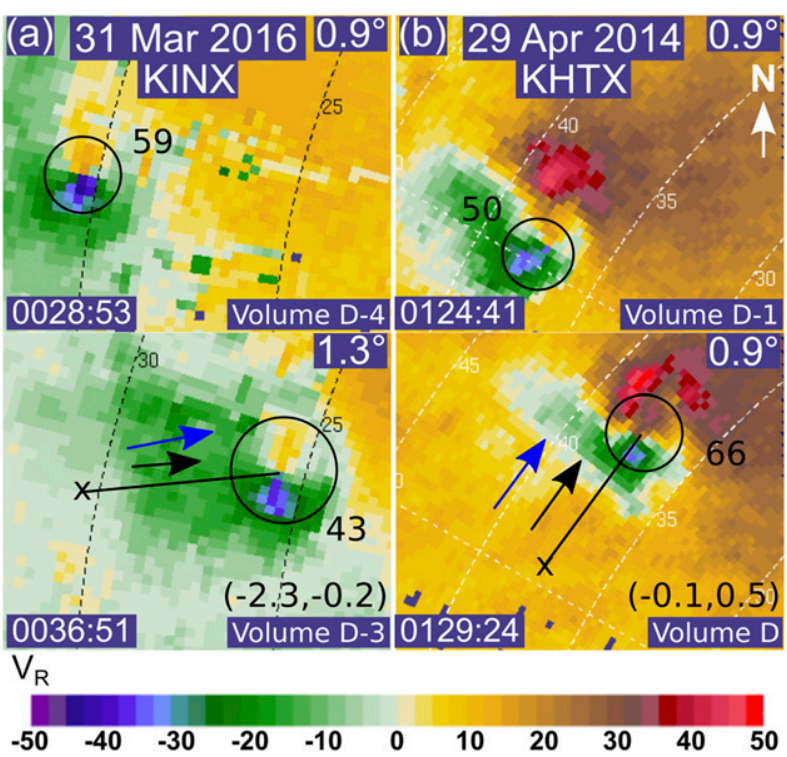

FIG. 15. Radial velocity $\left(\mathrm{m} \mathrm{s}^{-1}\right)$ scans of TVSs from the WSR88D in (a) Tulsa, OK (KINX), on 31 Mar 2016 and (b) Huntsville, AL (KHTX), on 29 Apr 2014. The black circles outline the TVSs and the numbers next to the outlines are the estimates of TVS intensity via the maximum TVS $\Delta V\left(\mathrm{~m} \mathrm{~s}^{-1}\right)$. The $\times$ annotations mark the approximate volume-by-volume progressions of the TVSs and the black (blue) arrows indicate the estimated TVS (storm) motion directions for that volume. The lengths of the arrows are proportional to magnitudes, and the $(u, v)$ components of TVS SR motion $\left(\mathrm{m} \mathrm{s}^{-1}\right)$ are provided in the bottom-right corner of each panel. Range rings are every $5 \mathrm{~km}$. The heights of the centers of the radar beams at the locations of the TVSs vary from $\sim 475$ to $600 \mathrm{~m}$ ARL.

\section{f. Use of SAILS and MESO-SAILS to assess TVS dissipation behaviors}

As discussed in section 1, the main barrier to the inclusion of more tornado dissipation cases using the WSR-88D network in this study is the lack of adequate temporal sampling of tornadoes. However, in recent years the implementation of Supplemental Adaptive Intravolume Low-Level Scans (SAILS; Chrisman 2013), and more recently Multiple Elevation Scan Option for SAILS (MESO-SAILS; Chrisman 2014), leads to some cases having finer temporal resolution than $4.5-5 \mathrm{~min}$ at low elevation angles. For example, in the 6 May 2015 case (Fig. 5), the TVS intensity progressively decreased and the TVS slowed and moved toward the north after the D-3 volume at $0.9^{\circ}$ elevation angle; the evolution at $0.5^{\circ}$ elevation angle was similar (Figs. 16a,c,e). With an additional scan at low levels per volume (Figs. 16b,d,f), there are three successive scans of TVS weakening and larger TVS SR motion over a $\sim 5$-min period rather than a 10-min period, effectively doubling the time resolution for nowcasting purposes. However, the shorter time between scans increases the likelihood of observed short-time-scale fluctuations in dissipation behaviors (e.g., the sharp change in TVS motion from Fig. 16b to Fig. 16c) owing to errors in TVS location and/or noise in the data that is amplified in time-derived quantities. In addition, TVS tilt and TVS-updraft horizontal displacement rely both on low- and midlevel data, the latter of which does not benefit from SAILS or MESO-SAILS, and would instead rely on increased use of advection correction over longer volume scan times. As a result, it is likely they do not benefit from changes in radar sampling at the lowest elevation angles alone.

\section{Summary and discussion}

There were two goals in studying TVS dissipation behaviors: 1) to determine better if previously identified tornado dissipation behaviors from observational case studies are consistent with TVS behaviors in a larger sample of cases and 2) to explore whether there are identifiable TVS dissipation behaviors that may serve as predictors of tornado dissipation in an operational setting.

Regarding the first goal, weaker and decreasing TVS intensity, rearward SR TVS motion, and large and increasing TVS-midlevel updraft horizontal displacement are shown to be associated with tornado dissipation at a statistically significant level; these results are consistent with past research from case studies. In addition, there is some evidence that rightward SR motion of dissipating tornadoes may be common in noncyclic supercells (French et al. 2014), though this conclusion is more tentative. There is no identified TVS tilt (inclination angle) associated with impending TVS dissipation in the lowest 2 or $3 \mathrm{~km}$, though there is perhaps a weak signal of increasing TVS tilt as TVSs dissipate. Also, this study did not address hypothesized causes for the four TVS behaviors (section 1), so we resist drawing clear relationships between tornado processes and TVS observations. ${ }^{5}$ We encourage the use of in-depth case studies using simulations or high-resolution data from mobile Doppler radars to test hypotheses related to mechanisms leading to tornado dissipation.

Regarding the second goal, we believe an important possibility for the research-to-operations community to

\footnotetext{
${ }^{5}$ For example, another potential contributor to SR TVS motion and TVS-midlevel updraft displacement is the possibility that some tornadoes may rotate about the mesocyclone (e.g., Fujita 1963; Wakimoto et al. 2003). In ground-relative TVS paths of the cases used in this study (not shown), there were a subset with a "kink" in their paths that would be consistent with this behavior (Wood 2016). However, using WSR-88D data to isolate how multiple processes may interact to influence an observed behavior is not feasible.
} 


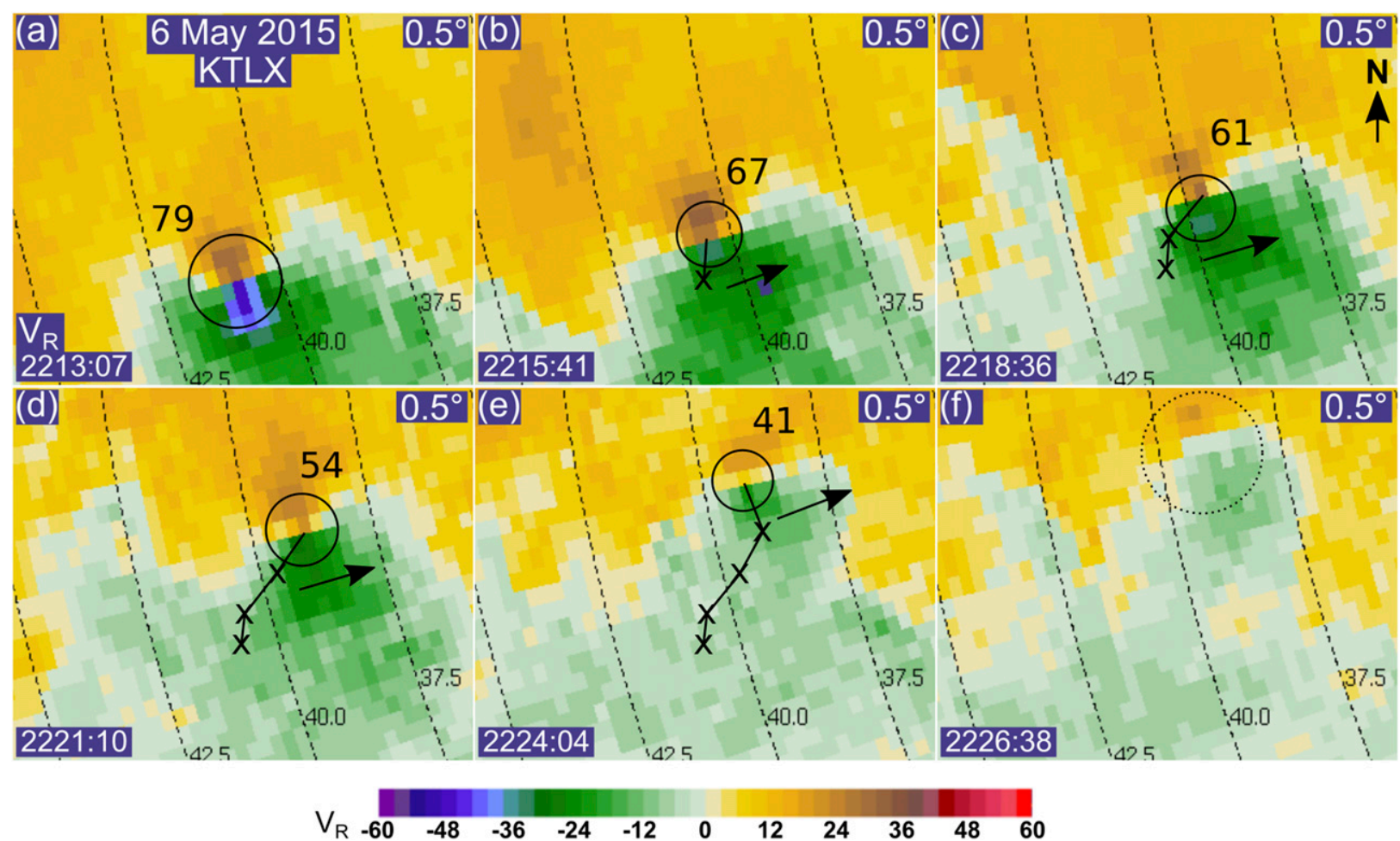

FIG. 16. As in Fig. 5, but at 0.5 elevation angle from (a) 2213:07, (b) 2215:41, (c) 2218:36, (d) 2221:10, (e) 2224:04, and (f) 2226:38 UTC. Range rings are every $2.5 \mathrm{~km}$. The height of the center of the radar beam at the location of the TVS is $\sim 350 \mathrm{~m}$ ARL. The storm motions in (a) and (b), in (c) and (d), and in (e) and (f) are identical because the scans in those groups come from the same volume.

consider is the use of radar observations in nowcasting tornado life cycles. Several of the TVS behaviors, and particularly combinations of behaviors, are seen in most cases just prior to TVS dissipation. Both the intensity and SR motion behaviors are identifiable at 1-km height levels (in addition to $500 \mathrm{~m}$ ), which could allow for the range of TVSs from WSR-88D sites to be increased from the $60-\mathrm{km}$ threshold range criterion used in this study. However, given the dearth of tornadoes that can be analyzed in real time for an extended duration, and the likely improvements that come about when SAILS and MESO-SAILS are implemented, such an endeavor likely would have to wait for a future operational radar network that includes volumetric rapid-scan capabilities (e.g., Zrnic et al. 2006). An upgraded future radar network may also allow for additional tornado dissipation behaviors to be investigated using large samples of cases; for example, initial TVS weakening in the 1-2-km layer (French et al. 2014; Houser et al. 2015) and increases in hook echo median raindrop size (French et al. 2015). Finally, the upgrade in operational satellite spatiotemporal resolution afforded by GOES-16/17 (Schmit et al. 2017) introduces the possibility that other nearreal-time remote sensing features associated with tornado dissipation may be identified in the future.
Operationally, the ability to identify trends in TVS intensity already exist within the Advanced Weather Interactive Processing System used by the National Weather Service, and barriers for streamlined analysis of SR TVS motion are few. In order for a forecaster to quickly identify the distance of the TVS from the main storm updraft, a consistent and reliable objective methodology for updraft identification would need to be implemented. However, given the ongoing developmental polarimetric signature work (e.g., Snyder et al. 2015; Kingfield and Picca 2018), the implementation of this type of product does not, in our opinion, have major intellectual or practical roadblocks. Radar nowcasting would ideally supplement real-time assessment of the near-storm environment, short-term modeling guidance, and efforts like Warn-On Forecast (Stensrud et al. 2009) and Forecasting a Continuum of Environmental Threats (Rothfusz et al. 2018), in order to best evaluate the most likely future state of an ongoing tornado.

While efforts to understand causes for tornado dissipation continue, we emphasize that its dynamical complexity and sensitivity to environmental heterogeneities at what are typically unobservable scales do not necessarily prevent its effects from being leveraged in a way that makes skillful tornado dissipation prediction realistic. Future 
improvements to U.S. operational remote sensing infrastructure, including GOES-16/17 and a potential phased array radar network upgrade, will provide opportunities for future work to both more explicitly identify dissipation behaviors and experiment with implementing observationally based tornado life cycle nowcasts.

Acknowledgments. This work was supported by NASA Grant NNX15AQ59G and NSF Grants AGS-1748177 and AGS-1748191. The authors thank Yvette Richardson (The Pennsylvania State University) for suggestions that substantially improved this study, and three anonymous reviewers for many helpful improvements. Joseph Clark (The Pennsylvania State University) worked with the first author on an earlier iteration of this project.

\section{REFERENCES}

Adlerman, E. J., K. K. Droegemeier, and R. Davies-Jones, 1999: A numerical simulation of cyclic mesocyclogenesis. J. Atmos. Sci., 56, 2045-2069, https://doi.org/10.1175/1520-0469(1999)056<2045: ANSOCM $>2.0 . \mathrm{CO} ; 2$.

Alexander, C. R., 2010: A mobile radar based climatology of supercell tornado structure and dynamics. Ph.D. dissertation, University of Oklahoma, $229 \mathrm{pp}$.

—_, and J. Wurman, 2005: The 30 May 1998 Spencer, South Dakota, storm. Part I: The structural evolution and environment of the tornadoes. Mon. Wea. Rev., 133, 72-97, https://doi.org/ 10.1175/MWR-2855.1.

Atkins, N. T., K. M. Butler, K. R. Flynn, and R. M. Wakimoto, 2014: An integrated damage, visual, and radar analysis of the 2013 Moore, Oklahoma, EF5 tornado. Bull. Amer. Meteor. Soc., 95, 1549-1561, https://doi.org/10.1175/BAMS-D-14-00033.1.

Blair, S. F., and J. W. Leighton, 2014: Assessing real-time tornado information disseminated through NWS products. Wea. Forecasting, 29, 591-600, https://doi.org/10.1175/WAF-D-1300126.1.

Bluestein, H. B., E. W. McCaul, G. P. Byrd, and G. R. Woodall, 1988: The unusual dissipation of a tornado funnel. Mon. Wea. Rev., 116, 950-952, https://doi.org/10.1175/1520-0493(1988)116<0950: TUDOAT $>2.0 . \mathrm{CO} ; 2$.

— C. C. Weiss, and A. L. Pazmany, 2003: Mobile Doppler radar observations of a tornado in a supercell near Bassett, Nebraska, on 5 June 1999. Part I: Tornadogenesis. Mon. Wea. Rev., 131, 2954-2967, https://doi.org/10.1175/1520-0493(2003)131<2954: MDROOA $>2.0 . \mathrm{CO} ; 2$.

—, M. M. French, J. C. Snyder, and J. B. Houser, 2016: Doppler radar observations of anticyclonic tornadoes in cyclonically rotating, right-moving supercells. Mon. Wea. Rev., 144, 15911616, https://doi.org/10.1175/MWR-D-15-0304.1.

Bodine, D. J., M. R. Kumjian, R. D. Palmer, P. L. Heinselman, and A. V. Ryzhkov, 2013: Tornado damage estimation using polarimetric radar. Wea. Forecasting, 28, 139-158, https://doi.org/ 10.1175/WAF-D-11-00158.1.

Brandes, E. A., 1977: Gust front evolution and tornado genesis as viewed by Doppler radar. J. Appl. Meteor., 16, 333-338, https://doi.org/ 10.1175/1520-0450(1977)016<0333:GFEATG >2.0.CO;2.

_ 1978: Mesocyclone evolution and tornadogenesis: Some observations. Mon. Wea. Rev., 106, 995-1011, https://doi.org/ 10.1175/1520-0493(1978)106<0995:MEATSO>2.0.CO;2.
- 1981: Fine structure of the Del City-Edmond tornadic mesocirculation. Mon. Wea. Rev., 109, 635-647, https://doi.org/ 10.1175/1520-0493(1981)109<0635:FOTDCE > 2.0.CO;2.

- 1984: Vertical vorticity generation and mesocyclone sustenance in tornadic thunderstorms: The observational evidence. Mon. Wea. Rev., 112, 2253-2269, https://doi.org/10.1175/ 1520-0493(1984)112<2253:VVGAMS > 2.0.CO;2.

Brooks, H. E., 2004: On the relationship of tornado path length and width to intensity. Wea. Forecasting, 19, 310-319, https://oi.org/ 10.1175/1520-0434(2004)019<0310:OTROTP >2.0.CO;2.

Brown, R. A., 1998: Nomogram for aiding the interpretation of tornadic vortex signatures measured by Doppler radar. Wea. Forecasting, 13, 505-512, https://doi.org/10.1175/ 1520-0434(1998)013<0505:NFATIO > 2.0.CO;2.

- and V. T. Wood, 2015: Detection of the presence of tornadoes at the center of mesocyclones using simulated Doppler velocity measurements. Wea. Forecasting, 30, 957-963, https:// doi.org/10.1175/WAF-D-15-0014.1.

— , L. R. Lemon, and D. W. Burgess, 1978: Tornado detection by pulsed Doppler radar. Mon. Wea. Rev., 106,29-39, https:// doi.org/10.1175/1520-0493(1978)106<0029:TDBPDR>2.0.CO;2.

Burgess, D. W., V. T. Wood, and R. A. Brown, 1982: Mesocyclone evolution statistics. Preprints, 12th Conf. on Severe Local Storms, San Antonio, TX, Amer. Meteor. Soc., 422-424.

— R. R. Donaldson Jr., and P. R. Desrochers, 1993: Tornado detection and warning by radar. The Tornado: Its Structure, Dynamics, Prediction, and Hazards, Geophys. Monogr., No. 79, Amer. Geophys. Union, 203-221.

_- M. A. Magsig, J. Wurman, D. C. Dowell, and Y. Richardson, 2002: Radar observations of the 3 May 1999 Oklahoma City tornado. Wea. Forecasting, 17, 456-471, https://doi.org/10.1175/ 1520-0434(2002)017<0456:ROOTMO > 2.0.CO;2.

Chrisman, J. N., 2013: Dynamic scanning. NEXRAD Now, No. 22, WSR-88D Radar Operations Center, Norman, OK, 1-3, https:// www.roc.noaa.gov/WSR88D/PublicDocs/NNOW/NNow22c.pdf.

_ 2014: Multiple Elevation Scan Option for SAILS (MESOSAILS) - The next step in dynamic scanning for the WSR88D. WSR-88D Radar Operations Center, 27 pp., https:// www.roc.noaa.gov/WSR88D/PublicDocs/NewTechnology/ MESO-SAILS_Description_Briefing_Jan_2014.pdf.

Coleman, T. A., and P. G. Dixon, 2014: An objective analysis of tornado risk in the United States. Wea. Forecasting, 29, 366376, https://doi.org/10.1175/WAF-D-13-00057.1.

Dahl, J. M. L., M. D. Parker, and L. J. Wicker, 2014: Imported and storm-generated near-ground vertical vorticity in a simulated supercell. J. Atmos. Sci., 71, 3027-3051, https://doi.org/10.1175/ JAS-D-13-0123.1.

Darkow, G. L., and J. C. Roos, 1970: Multiple tornado producing thunderstorms and their apparent cyclic variations in intensity. Preprints, 14th Conf. on Radar Meteorology, Tucson, AZ, Amer. Meteor. Soc., 305-308.

Davies-Jones, R. P., 1982: Observational and theoretical aspects of tornadogenesis. Intense Atmospheric Vortices, L. Bengtsson and J. Lighthill, Eds., Springer-Verlag, 175-189.

_, and H. E. Brooks, 1993: Mesocyclogenesis from a theoretical perspective. The Tornado: Its Structure, Dynamics, Prediction, and Hazards, Geophys. Monogr., Vol. 79, Amer. Geophys. Union, 105-114.

—, D. W. Burgess, L. R. Lemon, and D. Purcell, 1978: Interpretation of surface marks and debris patterns from the 24 May 1973 Union City, Oklahoma tornado. Mon. Wea. Rev., 106, 12-21, https://doi.org/10.1175/1520-0493(1978)106<0012: IOSMAD $>2.0 . \mathrm{CO} ; 2$. 
Dowell, D. C., and H. B. Bluestein, 2002a: The 8 June 1995 McLean, Texas, storm. Part I: Observations of cyclic tornadogenesis. Mon. Wea. Rev., 130, 2626-2648, https://doi.org/ 10.1175/1520-0493(2002)130<2626:TJMTSP > 2.0.CO;2.

- and _ 2002b: The 8 June 1995 McLean, Texas, storm. Part II: Cyclic tornado formation, maintenance, and dissipation. Mon. Wea. Rev., 130, 2649-2670, https://doi.org/10.1175/ 1520-0493(2002)130<2649:TJMTSP > 2.0.CO;2.

French, M. M., H. B. Bluestein, D. C. Dowell, L. J. Wicker, M. R. Kramar, and A. L. Pazmany, 2008: High-resolution, mobile Doppler radar observations of cyclic mesocyclogenesis in a supercell. Mon. Wea. Rev., 136, 4997-5016, https://doi.org/ 10.1175/2008MWR2407.1.

,-- I. IopStefanija, C. Baldi, and R. T. Bluth, 2013: Reexamining the vertical development of tornadic vortex signature in supercells. Mon. Wea. Rev., 141, 4576-4601, https://doi.org/ 10.1175/MWR-D-12-00315.1.

$\longrightarrow,-,-, \ldots$, and ——, 2014: Mobile, phased-array, Doppler radar observations of tornadoes at X band. Mon. Wea. Rev., 142, 1010-1036, https://doi.org/10.1175/MWR-D-13-00101.1.

, D. W. Burgess, E. R. Mansell, and L. J. Wicker, 2015: Bulk hook echo raindrop sizes retrieved using mobile, polarimetric Doppler radar observations. J. Appl. Meteor. Climatol., 54, 423-450, https://doi.org/10.1175/JAMC-D-14-0171.1.

Fujita, T. T., 1963: Analytical mesometeorology: A review. Severe Local Storms, Meteor. Monogr., No. 27, Amer. Meteor. Soc., 77-125.

—, D. L. Bradbury, and C. F. Van Thullenar, 1970: Palm Sunday tornadoes of April 11, 1965. Mon. Wea. Rev., 98, 29-69, https:// doi.org/10.1175/1520-0493(1970)098<0029:PSTOA > 2.3.CO;2.

Golden, J. H., and D. Purcell, 1977: Photogrammetric velocities for the Great Bend, Kansas, tornado of 30 August 1974: Accelerations and asymmetries. Mon. Wea. Rev., 105, 485-492, https://doi.org/ 10.1175/1520-0493(1977)105<0485:PVFTGB > 2.0.CO;2.

_ tornado and comparison with waterspouts. Mon. Wea. Rev., 106, 3-11, https://doi.org/10.1175/1520-0493(1978)106<0003: LCOTUC $>2.0 . \mathrm{CO} ; 2$.

Guarriello, F., C. Nowotarski, and C. Epifanio, 2018: Effects of the low-level wind profile on outflow position and near-surface vertical vorticity in simulated supercell thunderstorms. J. Atmos. Sci., 75, 731-753, https://doi.org/10.1175/JAS-D-17-0174.1.

Houser, J. L., H. B. Bluestein, and J. C. Snyder, 2015: Rapid-scan, polarimetric, Doppler radar observations of tornadogenesis and tornado dissipation in a tornadic supercell: The "El Reno, Oklahoma" storm of 24 May 2011. Mon. Wea. Rev., 143, 26852710, https://doi.org/10.1175/MWR-D-14-00253.1.

Illingworth, A. J., J. W. F. Goddard, and S. M. Cherry, 1987: Polarization radar studies of precipitation development in convective storms. Quart. J. Roy. Meteor. Soc., 113, 469-489, https://doi.org/10.1002/qj.49711347604.

Kingfield, D. M., and J. G. LaDue, 2015: The relationship between automated low-level velocity calculations from the WSR-88D and maximum tornado intensity determined from damage surveys. Wea. Forecasting, 30, 1125-1139, https://doi.org/ 10.1175/WAF-D-14-00096.1.

— , and J. C. Picca, 2018: Development of an operational convective nowcasting algorithm using raindrop size sorting information from polarimetric radar data. Wea. Forecasting, 33, 1477-1495, https://doi.org/10.1175/WAF-D-18-0025.1.

Klees, A. M., Y. P. Richardson, P. M. Markowski, C. Weiss, J. M. Wurman, and K. K. Kosiba, 2016: Comparison of the tornadic and nontornadic supercells intercepted by VORTEX2 on
10 June 2010. Mon. Wea. Rev., 144, 3201-3231, https://doi.org/ 10.1175/MWR-D-15-0345.1.

Kosiba, K. A., J. Wurman, Y. Richardson, P. Markowski, P. Robinson, and J. Marquis, 2013: Genesis of the Goshen County, Wyoming, tornado on 5 June 2009 during VORTEX2. Mon. Wea. Rev., 141, 1157-1181, https://doi.org/10.1175/MWR-D-12-00056.1.

Kumjian, M. R., and A. V. Ryzhkov, 2008: Polarimetric signatures in supercell thunderstorms. J. Appl. Meteor. Climatol., 47, 1940-1961, https://doi.org/10.1175/2007JAMC1874.1.

_ A. P. Khain, N. Benmoshe, E. Ilotoviz, A. V. Ryzhkov, and V. T. J. Phillips, 2014: The anatomy and physics of $Z_{\mathrm{DR}}$ columns: Investigating a polarimetric radar signature with a spectral bin microphysical model. J. Appl. Meteor. Climatol., 53, 1820-1843, https://doi.org/10.1175/JAMC-D-13-0354.1.

Kurdzo, J. M., D. J. Bodine, B. L. Cheong, and R. D. Palmer, 2015: High-temporal resolution polarimetric X-band Doppler radar observations of the 20 May 2013 Moore, Oklahoma, tornado. Mon. Wea. Rev., 143, 2711-2735, https://doi.org/10.1175/ MWR-D-14-00357.1.

Lee, W.-C., and J. Wurman, 2005: Diagnosed three-dimensional axisymmetric structure of the Mulhall tornado on 3 May 1999. J. Atmos. Sci., 62, 2373-2393, https://doi.org/10.1175/ JAS3489.1.

Lemon, L. R., and C. A. Doswell, 1979: Severe thunderstorm evolution and mesocyclone structure as related to tornadogenesis. Mon. Wea. Rev., 107, 1184-1197, https://doi.org/ 10.1175/1520-0493(1979)107<1184:STEAMS > 2.0.CO;2.

Markowski, P. M., and Y. P. Richardson, 2009: Tornadogenesis: Our current understanding, forecasting considerations, and questions to guide future research. Atmos. Res., 93, 3-10, https://doi.org/10.1016/j.atmosres.2008.09.015.

— shear and cold pools on tornadogenesis: Insights from idealized simulations. J. Atmos. Sci., 71, 243-275, https://doi.org/ 10.1175/JAS-D-13-0159.1.

_ J. M. Straka, and E. N. Rasmussen, 2002: Direct surface thermodynamic observations within the rear-flank downdrafts of nontornadic and tornadic supercells. Mon. Wea. Rev., 130, 1692-1721, https://doi.org/10.1175/1520-0493(2002)130<1692: DSTOWT $>2.0$.CO;2.

_- Y. Richardson, M. Majcen, J. Marquis, and J. Wurman, 2011: Characteristics of the wind field in three nontornadic low-level mesocyclones observed by the Doppler on Wheels radars. Electron. J. Severe Storms Meteor., 6 (3), http://www.ejssm.org/ ojs/index.php/ejssm/article/viewArticle/75.

—_, J. Marquis, J. Wurman, K. Kosiba, P. Robinson, E. Rasmussen, and D. Dowell, 2012: The pretornadic phase of the Goshen County, Wyoming, supercell of 5 June 2009 intercepted by VORTEX2. Part II: Intensification of low-level rotation. Mon. Wea. Rev., 140, 2916-2938, https://doi.org/ 10.1175/MWR-D-11-00337.1.

Marquis, J., Y. Richardson, J. Wurman, and P. Markowski, 2008: Single- and dual- Doppler analysis of a tornadic vortex and surrounding storm-scale flow in the Crowell, Texas, supercell of 30 April 2000. Mon. Wea. Rev., 136, 5017-5043, https://doi.org/ 10.1175/2008MWR2442.1.

— - _ P. Markowski, D. Dowell, and J. Wurman, 2012: Tornado maintenance investigated with high-resolution dual-Doppler and EnKF analysis. Mon. Wea. Rev., 140, 3-27, https://doi.org/10.1175/MWR-D-11-00025.1.

Pratt, J., 1959: Remarks on zeros and ties in the Wilcoxon signed rank procedures. J. Amer. Stat. Assoc., 54, 655-667, https://doi.org/ 10.1080/01621459.1959.10501526. 
Rothfusz, L. P., R. Schneider, D. Novak, K. Klockow, A. E. Gerard, C. Karstens, G. J. Stumpf, and T. M. Smith, 2018: FACETs: A proposed next-generation paradigm for highimpact weather forecasting. Bull. Amer. Meteor. Soc., 99, 2025-2043, https://doi.org/10.1175/BAMS-D-16-0100.1.

Ryzhkov, A., T. J. Schuur, D. W. Burgess, and D. S. Zrnić, 2005: Polarimetric tornado detection. J. Appl. Meteor., 44, 557-570, https://doi.org/10.1175/JAM2235.1.

Schmit, T. J., P. Griffith, M. M. Gunshor, J. M. Daniels, S. J. Goodman, and W. J. Lebair, 2017: A closer look at the ABI on the GOES-R series. Bull. Amer. Meteor. Soc., 98, 681-698, https://doi.org/10.1175/BAMS-D-15-00230.1.

Skinner, P. S., C. C. Weiss, M. M. French, H. B. Bluestein, P. M. Markowski, and Y. P. Richardson, 2014: VORTEX2 observations of a low-level mesocyclone with multiple internal rearflank downdraft momentum surges in the 18 May 2010 Dumas, Texas, supercell. Mon. Wea. Rev., 142, 2935-2960, https:// doi.org/10.1175/MWR-D-13-00240.1.

Snyder, J. C., and H. B. Bluestein, 2014: Some considerations for the use of high-resolution mobile radar data in tornado intensity determination. Wea. Forecasting, 29, 799-827, https:// doi.org/10.1175/WAF-D-14-00026.1.

— , and A. V. Ryzhkov, 2015: Automated detection of polarimetric tornadic debris signatures using a hydrometeor classification algorithm. J. Appl. Meteor. Climatol., 54, 1861-1870, https://doi.org/10.1175/JAMC-D-15-0138.1.

,-- M. R. Kumjian, A. P. Khain, and J. Picca, 2015: A $Z_{\mathrm{DR}}$ column detection algorithm to examine convective storm updrafts. Wea. Forecasting, 30, 1819-1844, https://doi.org/ 10.1175/WAF-D-15-0068.1.

Speheger, D. A., C. A. Doswell, and G. J. Stumpf, 2002: The tornadoes of 3 May 1999: Event verification in central Oklahoma and related issues. Wea. Forecasting, 17, 362-381, https://doi.org/ 10.1175/1520-0434(2002)017<0362:TTOMEV >2.0.CO;2.

Stensrud, D. J., and Coauthors, 2009: Convective-scale warn-onforecast system: A vision for 2020. Bull. Amer. Meteor. Soc., 90, 1487-1499, https://doi.org/10.1175/2009BAMS2795.1.

Tanamachi, R. L., H. B. Bluestein, J. B. Houser, S. J. Frasier, and K. M. Hardwick, 2012: Mobile, X-band, polarimetric Doppler radar observations of the 4 May 2007 Greensburg, Kansas, tornadic supercell. Mon. Wea. Rev., 140, 2103-2125, https:// doi.org/10.1175/MWR-D-11-00142.1.

,-- M. Xue, W.-C. Lee, K. A. Orzel, S. J. Frasier, and R. M. Wakimoto, 2013: Near-surface vortex structure in a tornado and in a sub-tornado-strength convective-storm vortex observed by a mobile, W-Band radar during VORTEX2. Mon. Wea. Rev., 141, 3661-3690, https://doi.org/10.1175/MWR-D-12-00331.1.

Torres, S. M., and C. D. Curtis, 2007: Initial implementation of super-resolution data on the NEXRAD network. Preprints, 23rd Conf. on Information Processing Systems for Meteorology, Oceanography, and Hydrology, San Antonio, TX, Amer. Meteor. Soc., 5B.10, https://ams.confex.com/ams/pdfpapers/ 116240.pdf.

Trapp, R. J., E. D. Mitchell, G. A. Tipton, D. W. Effertz, A. I. Watson, D. L. Andra, and M. A. Magsig, 1999: Descending and nondescending tornadic vortex signatures detected by WSR-88Ds. Wea. Forecasting, 14, 625-639, https://doi.org/ 10.1175/1520-0434(1999)014<0625:DANTVS>2.0.CO;2.

Wakimoto, R. M., and B. E. Martner, 1992: Observations of a Colorado tornado. Part II: Combined photogrammetric and Doppler radar analysis. Mon. Wea. Rev., 120, 522-543, https://doi.org/ 10.1175/1520-0493(1992)120<0522:OOACTP >2.0.CO;2.

, H. V. Murphey, D. C. Dowell, and H. B. Bluestein, 2003: The Kellerville tornado during VORTEX: Damage survey and Doppler radar analyses. Mon. Wea. Rev., 131, 2197-2221, https:// doi.org/10.1175/1520-0493(2003)131<2197:TKTDVD>2.0.CO;2.

Wicker, L. J., and R. B. Wilhelmson, 1995: Simulation and analysis of tornado development and decay within a three-dimensional supercell thunderstorm. J. Atmos. Sci., 52, 2675-2703, https:// doi.org/10.1175/1520-0469(1995)052<2675:SAAOTD>2.0.CO;2.

Wilcoxon, F., 1945: Individual comparisons by ranking methods. Biom. Bull., 1, 80-83, https://doi.org/10.2307/3001968.

Witt, A., M. D. Eilts, G. J. Stumpf, E. D. Mitchell, J. T. Johnson, and K. W. Thomas, 1998: Evaluating the performance of WSR-88D severe storm detection algorithms. Wea. Forecasting, 13, 513-518, https://doi.org/10.1175/1520-0434(1998)013<0513: ETPOWS $>2.0$.CO;2.

Wood, V. T., 2016: Trochoidal paths traced out by a subvortex revolving around a parent vortex: A simulation study. 28th Conf. on Severe Local Storms, Portland, OR, Amer. Meteor. Soc., P35, https://ams.confex.com/ams/28SLS/webprogram/ Paper299175.html.

— , and R. A. Brown, 1997: Effects of radar sampling on singleDoppler velocity signatures of mesocyclones and tornadoes. Wea. Forecasting, 12, 928-938, https://doi.org/10.1175/ 1520-0434(1997)012<0928:EORSOS>2.0.CO;2.

$\longrightarrow$, and -2011 : Simulated tornadic vortex signatures of tornado-like vortices having one- and two-celled structures. J. Appl. Meteor. Climatol., 50, 2338-2342, https://doi.org/ 10.1175/JAMC-D-11-0118.1.

$\longrightarrow,-$, and D. C. Dowell, 2009: Simulated WSR-88D velocity and reflectivity signatures of numerically modelled tornadoes. J. Atmos. Oceanic Technol., 26, 876-893, https://doi.org/ 10.1175/2008JTECHA1181.1.

Wurman, J., and S. Gill, 2000: Finescale radar observations of the Dimmitt, Texas (2 June 1995), tornado. Mon. Wea. Rev., 128, 2135-2164, https://doi.org/10.1175/1520-0493(2000)128<2135: FROOTD $>2.0 . \mathrm{CO} ; 2$.

- and K. Kosiba, 2013: Finescale radar observations of tornado and mesocyclone structures. Wea. Forecasting, 28, 1157-1174, https://doi.org/10.1175/WAF-D-12-00127.1.

-, Y. Richardson, C. Alexander, S. Weygandt, and P. Zhang, 2007a: Dual-Doppler and single-Doppler analysis of a tornadic storm undergoing mergers and repeated tornadogenesis. Mon. Wea. Rev., 135, 736-758, https://doi.org/10.1175/MWR3276.1.

,,,,---- and,$- 2007 \mathrm{~b}$ : Dual-Doppler analysis of winds and vorticity budget terms near a tornado. Mon. Wea. Rev., 135, 2392-2405, https://doi.org/10.1175/MWR3404.1.

, K. Kosiba, P. Markowski, Y. Richardson, D. Dowell, and P. Robinson, 2010: Finescale single- and dual-Doppler analysis of a tornado intensification, maintenance, and dissipation in the Orleans, Nebraska, supercell. Mon. Wea. Rev., 138, 4439-4455, https://doi.org/10.1175/2010MWR3330.1.

, D. Dowell, Y. Richardson, P. Markowski, E. Rasmussen, D. Burgess, L. Wicker, and H. B. Bluestein, 2012: The second Verification of the Origins of Rotation in Tornadoes Experiment: VORTEX2. Bull. Amer. Meteor. Soc., 93, 1147-1170, https://doi.org/10.1175/BAMS-D-11-00010.1.

Zrnic, D. S., V. M. Melnikov, and J. K. Carter, 2006: Calibrating differential reflectivity on the WSR-88D. J. Atmos. Oceanic Technol., 23, 944-951, https://doi.org/10.1175/JTECH1893.1. 